\title{
The NHL domain of BRAT is an RNA- binding domain that directly contacts the hunchback mRNA for regulation
}

\author{
Inga Loedige, ${ }^{1,8,9}$ Mathias Stotz, ${ }^{1,8}$ Saadia Qamar, ${ }^{2,3}$ Katharina Kramer, ${ }^{2,3}$ Janosch Hennig, ${ }^{4,5}$ \\ Thomas Schubert, ${ }^{6}$ Patrick Löffler, ${ }^{7}$ Gernot Längst, ${ }^{6}$ Rainer Merkl, ${ }^{7}$ Henning Urlaub, ${ }^{2,3}$ \\ and Gunter Meister ${ }^{1,9}$ \\ ${ }^{1}$ Biochemistry Center Regensburg (BZR), Laboratory for RNA Biology, University of Regensburg, 93053 Regensburg, Germany; \\ ${ }^{2}$ Bioanalytical Mass Spectrometry, Max Planck Institute for Biophysical Chemistry, 37077 Göttingen, Germany; ${ }^{3}$ Bioanalytics, \\ Institute for Clinical Chemistry, University Medical Center Göttingen, 37075 Göttingen, Germany; ${ }^{4}$ Group Biomolecular NMR, \\ Institute of Structural Biology, Helmholtz Zentrum München-German Research Center for Environmental Health, 85764 \\ Neuherberg, Germany; ${ }^{5}$ Center for Integrated Protein Science Munich, Biomolecular NMR, Department Chemie, Technische \\ Universität München, 85748 Garching, Germany; ${ }^{6}$ Institute for Biochemistry III, University of Regensburg, 93053 Regensburg, \\ Germany; ${ }^{7}$ Institute of Biophysics and Physical Biochemistry, University of Regensburg, 93053 Regensburg, Germany
}

The Drosophila protein brain tumor (Brat) forms a complex with Pumilio (Pum) and Nanos (Nos) to repress hunchback (hb) mRNA translation at the posterior pole during early embryonic development. It is currently thought that complex formation is initiated by Pum, which directly binds the hb mRNA and subsequently recruits Nos and Brat. Here we report that, in addition to Pum, Brat also directly interacts with the hb mRNA. We identify Brat-binding sites distinct from the Pum consensus motif and show that RNA binding and translational repression by Brat do not require Pum, suggesting so far unrecognized Pum-independent Brat functions. Using various biochemical and biophysical methods, we also demonstrate that the NHL (NCL-1, HT2A, and LIN-41) domain of Brat, a domain previously believed to mediate protein-protein interactions, is a novel, sequence-specific ssRNA-binding domain. The Brat-NHL domain folds into a six-bladed $\beta$ propeller, and we identify its positively charged top surface as the RNA-binding site. Brat belongs to the functional diverse TRIM (tripartite motif)-NHL protein family. Using structural homology modeling, we predict that the NHL domains of all TRIM-NHL proteins have the potential to bind RNA, indicating that Brat is part of a conserved family of RNA-binding proteins.

[Keywords: BRAT; RNA binding; TRIM-NHL; gene regulation; hunchback; translational repression]

Supplemental material is available for this article.

Received December 16, 2013; revised version accepted February 20, 2014.

Post-transcriptional gene regulation plays an important role during early development of many organisms. In Drosophila embryos, the genome is transcriptionally silent, and the differential localization, stabilization, and translation of maternally provided mRNAs generate protein gradients that specify the body axes (NussleinVolhard et al. 1987; Curtis et al. 1995). Translation of the maternally provided and, in the embryo, uniformly distributed hunchback (hb) mRNA, for example, is restricted to the anterior pole of the embryo, where it directs head and thorax formation (Tautz and Pfeifle 1989; Hulskamp et al. 1990). Aberrant expression of $\mathrm{Hb}$

\footnotetext{
${ }^{8}$ These authors contributed equally to this work.

${ }^{9}$ Corresponding authors

E-mail gunter.meister@vkl.uni-regensburg.de

E-mail inga.loedige@vkl.uni-regensburg.de

Article is online at http://www.genesdev.org/cgi/doi/10.1101/gad.236513.113.
}

protein at the posterior pole, on the contrary, disrupts abdominal segmentation.

Inhibition of hb translation at the posterior pole requires a repressive complex containing the proteins Pumilio (Pum), Nanos (Nos), and brain tumor (Brat) and two regulatory elements, referred to as the Nanos response elements (NREs), located in the $3^{\prime}$ untranslated region (UTR) of the hb mRNA (Wharton and Struhl 1991; Murata and Wharton 1995; Sonoda and Wharton 2001). The NREs are high-affinity binding sites for the sequencespecific RNA-binding protein Pum (Murata and Wharton

(C) 2014 Loedige et al. This article is distributed exclusively by Cold Spring Harbor Laboratory Press for the first six months after the full-issue publication date (see http://genesdev.cshlp.org/site/misc/terms.xhtml). After six months, it is available under a Creative Commons License (Attribution-NonCommercial 4.0 International), as described at http:// creativecommons.org/licenses/by-nc/4.0/. 
1995; Zamore et al. 1997), which further recruits Nos and Brat to the complex (Sonoda and Wharton 1999, 2001). While Nos determines the site and extent of hb repression (by a mechanism that is not yet fully understood), Brat interacts with the cap-binding protein d4EHP to inhibit translational initiation (Cho et al. 2006).

Apart from the regulation of the hb mRNA during early embryogenesis, several additional examples of translational regulation by a repressive complex of Brat and Pum or Brat, Pum, and Nos in various developmental contexts have been reported. For example, Brat and Pum repress myc and mad translation to promote differentiation during oogenesis (Harris et al. 2011). Furthermore, Brat, Pum, and Nos inhibit the paralytic mRNA to control excitability in motorneurons (Muraro et al. 2008), and, finally, in neuromuscular synapses, repression of mad mRNA by Brat regulates synaptic size (Shi et al. 2013).

Brat belongs to the conserved family of tripartite motif (TRIM)-NHL (NCL-1, HT2A, and LIN-41), which are characterized by their N-terminal TRIM and a C-terminal NHL domain (Sardiello et al. 2008). The TRIM motif consists of a RING domain, conferring ubiquitin or ubiquitin-like ligase activity, followed by one or two B-box motifs and a coiled coil region. Brat, however, is only an "incomplete" TRIM, as it lacks the RING domain (Fig. 1A). The NHL domain, which is named after the three proteins NCL-1, HT2A (TRIM32), and LIN-41 in which it was first identified (Slack and Ruvkun 1998), is of critical importance for Brat function: Flies carrying a deletion of or single point mutations within the NHL domain are characterized by various mutant phenotypes, including abdominal segmentation defects that can be rescued by re-expression of the wild-type NHL domain alone (Arama et al. 2000; Sonoda and Wharton 2001).

The crystal structure of the Brat-NHL domain revealed a six-bladed $\beta$ propeller, a fold resembling that of WD40 domains (Edwards et al. 2003). Owing to their large surfaces, $\beta$-propeller structures often provide platforms for multiple protein-protein interactions and often serve as hubs in cellular protein interaction networks (Stirnimann et al. 2010). Consistent with this idea, mutagenesis studies in flies showed that the top surface of the Brat-NHL domain interacts with Pum and is thus essential for the recruitment of Brat to RNA (Sonoda and Wharton 2001; Edwards et al. 2003), while its bottom surface contacts d4EHP (Cho et al. 2006).

In addition to its role as a translational repressor, Brat has been recognized as a powerful growth suppressor and differentiation factor (Frank et al. 2002; Betschinger et al. 2006; Lee et al. 2006; Harris et al. 2011). During asymmetric cell divisions (a characteristic hallmark of stem cells), Brat is confined to the differentiating daughter cell, where it restricts proliferation and promotes differentiation. Lack of Brat function causes tumorous overgrowth of the larval brain that is due to the failure of neuronal progenitor cells to exit proliferation (Betschinger et al. 2006; Lee et al. 2006). It is currently unclear whether the brain tumor phenotype is linked to Brat's function as a translational repressor, but post-transcriptional down- regulation of myc has been reported in this context (Betschinger et al. 2006). Strikingly, the same mutations that impair Brat's interaction with Pum and therefore disrupt Brat-mediated translational repression are also the cause of the overproliferation phenotype. A role for Pum in this process, however, has not been described.

Our previous work indicated that the mammalian TRIM-NHL protein TRIM71/LIN-41, which shares a similar domain architecture with Brat, acts as a translational repressor, and we identified its NHL domain as necessary and sufficient for RNA targeting (Loedige et al. 2013). A role for the mammalian Pum proteins PUM1 and PUM2 in mediating RNA binding of TRIM71, however, was not evident. In the meantime, a more global UV-cross-linking approach reported an RNA-binding activity of the TRIM71NHL domain (Kwon et al. 2013).

Using various biochemical and biophysical assays, we demonstrated that the NHL domain of Brat directly binds RNA with high affinity. We identified the BoxA motif of the NRE as the Brat-binding site and demonstrated that RNA binding and translational repression by Brat are independent of Pum. Using UV-cross-linking followed by mass spectrometry (MS), we identified the top surface of the NHL domain as the RNA-binding surface. Mutations of single residues on the top surface abrogate Brat-NHL RNA binding in vitro and impair translational repression by Brat in vivo.

\section{Results}

The NHL domain of Drosophila Brat directly binds $R N A$

An 100-nucleotide (nt)-long fragment from the hb 3' UTR that contains the two NRE's (termed hb RNA hereafter) (Fig. 1B) is required for translational repression of the hb mRNA in vivo (Wharton and Struhl 1991). The current model suggests that upon RNA binding, Pum serves as a platform and recruits Nos and Brat to the RNA by protein-protein interactions (Sonoda and Wharton 1999, 2001). To gain insight into a putative RNA-binding activity of Brat, we performed electrophoretic mobility shift assays (EMSAs or band shift assays) using in vitro transcribed hb RNA as a substrate and the recombinantly expressed and purified NHL domain of Brat (encompassing amino acids 756-1037) (Supplemental Fig. 1A).

We incubated increasing amounts of recombinant BratNHL with the radiolabeled hb RNA and analyzed the resulting protein-RNA complexes by native gel electrophoresis (Fig. 1C). Indeed, the Brat-NHL domain readily bound RNA with high affinity: At a protein concentration of 100-150 nM, half of the free RNA was shifted into a slower-migrating RNA-protein complex (Fig. 1C, lanes 6,7; Supplemental Fig. 2B, indicated by a black arrow). With increasing protein concentrations, a second, less well-defined band appeared (Fig. 1C, lanes 9-13, Supplemental Fig. 2B, marked by an asterisk). RNA binding of the Brat-NHL domain was sequence-specific, as the labeled RNA-protein complexes could be chased by an excess of unlabeled hb RNA but not by an excess of tRNA that was 


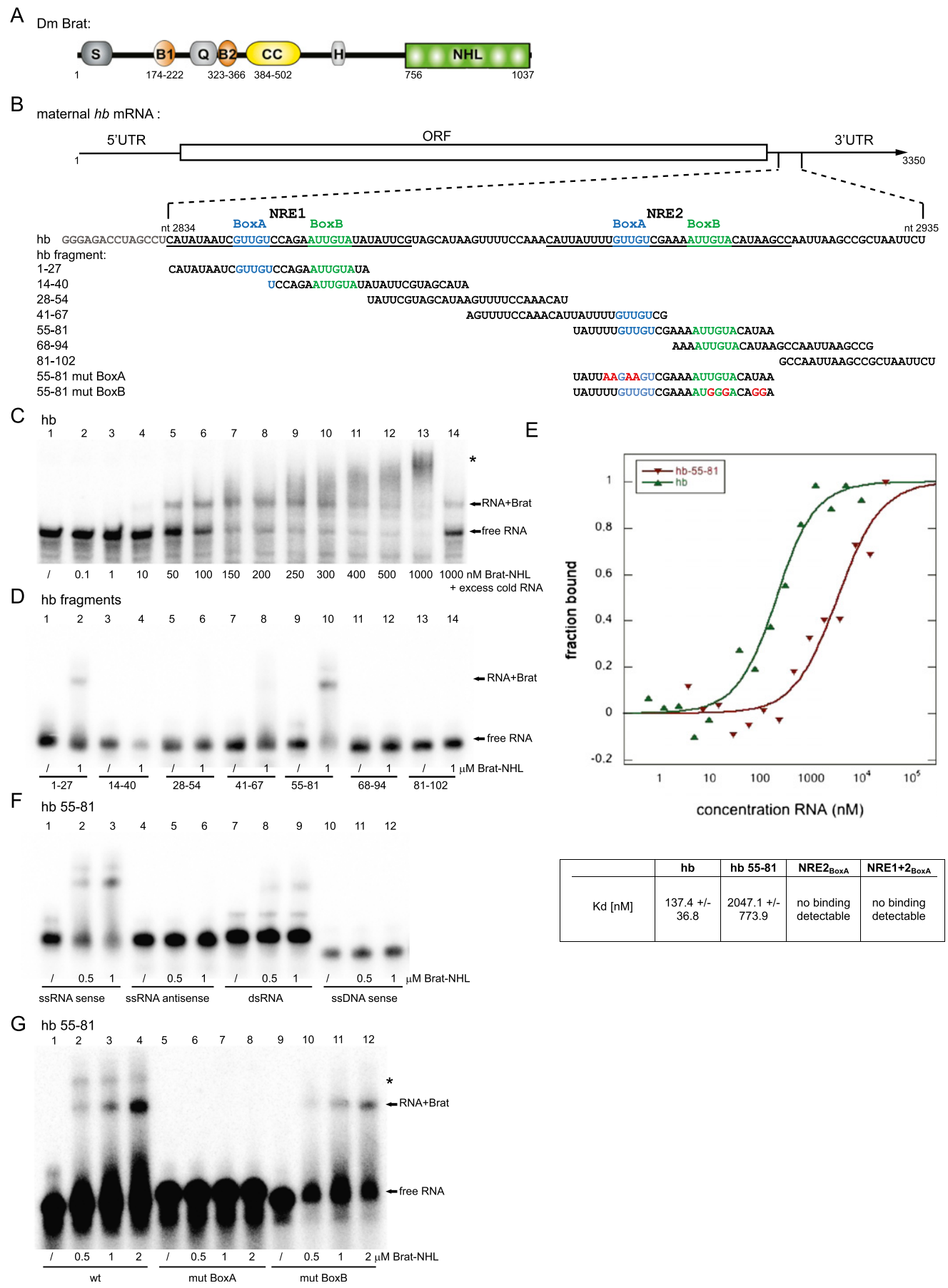

Figure 1. Direct RNA binding of the Brat-NHL domain. (A) Schematic representation of Drosophila melanogaster Brat domain organization. The two B-boxes (B1 and B2) are shown in orange, the coiled coil (CC) domain is in yellow, and the NHL domain, composed of six NHL repeats, is in green. Also indicated and colored in gray are serine-rich (S), glutamine-rich $(\mathrm{Q})$, and histidine-rich $(\mathrm{H})$ stretches. The numbers below indicate domain boundaries. (B) Schematic presentation of the maternally derived hb mRNA (NM_169234) and hb RNA fragments used in this study. The $\sim 100$-nt-long hb RNA fragment (for simplicity referred to here as hb RNA) contains two NREs, each composed of one BoxA and one BoxB motif. Nucleotides mutated in hb 55-81 are indicated by red letters. $(C)$ Native gel analysis to probe for RNA binding of the Brat-NHL domain. Increasing amounts of recombinant Brat-NHL (amino acids 756-1037) were incubated with 500 pM ${ }^{32} \mathrm{P}$-labeled hb RNA, and complexes were analyzed by native gel electrophoresis. $(D)$ Brat-NHL binds sequences that contain the NRE. Short, 27-nt, ${ }^{32} \mathrm{P}$-labeled RNA probes that span the hb RNA (as depicted in $A$ ) were incubated with the indicated amounts of recombinant BratNHL, and complexes were analyzed by native gel electrophoresis. (E) MST measurements. (Top) Representative binding curves for the interaction of Brat-NHL with hb RNA (green triangles) or hb fragment 55-81 (red triangles). (Bottom) Summary of independent MST measurements. Brat-NHL binds the hb RNA with a $K_{d}$ of $137.4 \mathrm{nM} \pm 36.8 \mathrm{nM}$ (six independent repeats); the short hb (fragment $55-81$ ) is bound with lower affinity and a $K_{d}$ of $2.0 \mu \mathrm{M} \pm 0.8 \mu \mathrm{M}$ (five independent repeats). No binding was detected for hb mutants that lack the Brat-binding motif (NRE2 $2_{\text {BoxA }}$ or NRE1+2 $2_{\text {BoxA }}$ ) (see Fig. 2). (F) The Brat-NHL domain is a sequence-specific, ssRNA-binding domain. Complex formation of recombinant Brat-NHL with ssRNA (lanes 1-3), ssRNA of antisense sequence (lanes 4-6), dsRNA (lanes 7-9), or ssDNA (lanes 10-12) was analyzed by native gel electrophoresis. In all cases, nucleotide sequences corresponding to hb fragment $55-81$ were used. DNA oligonucleotides contained dT instead of dU. $(G)$ Mutations in BoxA but not in BoxB abrogate Brat-NHL binding to the NRE2. Native gel analysis to test Brat-NHL binding to hb 55-81 or the indicated mutants. Free RNA or RNA-protein complexes are indicated by black arrows. Asterisk $\left({ }^{\star}\right)$ denotes a less well-defined Brat-NHL-hb RNA complex appearing at high protein concentrations. 
present in all reactions (Fig. 1C; Supplemental Fig. 2A). Our data therefore demonstrate that the NHL domain of Brat is able to bind to the hb RNA directly and in a sequence-specific manner.

\section{The Brat-NHL domain binds the NRE}

To determine which RNA sequences within the hb mRNA are responsible for Brat recruitment, we divided the 100-nt-long hb RNA used above into seven overlapping fragments of $27 \mathrm{nt}$ in length (Fig. 1B) and tested them in EMSAs (Fig. 1D). The Brat-NHL domain interacted with fragments that contained either NRE1 (fragment 1-27) or NRE2 (fragment 55-81) (Fig. 1D, lanes 2,10, respectively). Of note, binding of the Brat-NHL domain to these short fragments was much weaker than observed for the longer hb RNA, and a concentration of $2 \mu \mathrm{M}$ protein was needed to shift half of the short hb RNA (fragment 55-81) into a slower-migrating protein-RNA complex (Supplemental Fig. 2C).

To confirm our observations by an independent approach, we determined binding affinities using microscale thermophoresis (MST) (Fig. 1E), a technique that is based on the movement of molecules in temperature gradients (Jerabek-Willemsen et al. 2011; Zillner et al. 2012). The thermophoretic mobility of fluorescently labeled Brat-NHL was recorded at different RNA concentrations ranging from 0 to $10 \mu \mathrm{M}$ (long hb RNA) or $30 \mu \mathrm{M}$ (short hb RNA), and the data were used to calculate binding affinities (Fig. 1E). The determined binding affinities from independent thermophoresis experiments, each performed with independently labeled Brat-NHL, closely matched the affinities determined in our gel shift assays: The Brat-NHL domain bound to the long hb RNA with a $K_{d}$ of $137.4 \mathrm{nM} \pm 36.8 \mathrm{nM}$, while the shorter fragment encompassing NRE2 (hb 55-81) interacted with a $K_{d}$ of $2 \mu \mathrm{M} \pm 0.8 \mu \mathrm{M}$ (Fig. 1E). Hb RNA mutants that lack the Brat-binding motif, which is described in more detail below (Figs. 1G, 2), showed no detectable binding (Fig. 1E), confirming the validity of the assay.

\section{The Brat-NHL domain binds ssRNA}

To determine the substrate preference of the Brat-NHL domain, we analyzed Brat-NHL binding to ssRNA (hb 5581), ssRNA containing the antisense sequence, dsRNA, or ssDNA (Fig. 1F). All oligonucleotides were $27 \mathrm{nt}$ long and were based on the hb 55-81 fragment shown in Figure 1D. Brat-NHL bound to the single-stranded sense (Fig. 1D, lanes 2,3) but not the single-stranded antisense (Fig. 1D, lanes 5,6) sequence, again confirming sequencespecific binding. No binding was observed to ssDNA (Fig. $1 \mathrm{D}$, lanes 11,12 ), and only weak binding to dsRNA was detectable (Fig. 1D, lanes 8,9). It is conceivable that this weak binding is due to incomplete annealing of the two single strands and would thus reflect binding of the BratNHL domain to ssRNA.

\section{The Brat-NHL domain binds to BoxA of the hb NRE}

Each of the two NREs is composed of one BoxA and one BoxB motif, both of which contribute to hb mRNA regulation in vivo (Murata and Wharton 1995). While the BoxB motif constitutes the high-affinity binding site for Pum (Zamore et al. 1997; Wang et al. 2002), the exact contribution of the BoxA motif to hb regulation is unclear. To further define which sequence element within the NRE in the hb mRNA is responsible for Brat-NHL recruitment, we introduced mutations into either the short 27-nt hb RNA fragment encompassing NRE2 (hb 55-81) (Fig. 1G) or the long 100-nt hb RNA (Fig. 2) and determined their effect on Brat-NHL binding in band shift assays. Mutation of four uracils into adenines within or preceding the BoxA motif (hb 55-81 mut BoxA) abolished Brat-NHL binding to the short hb 55-81 fragment (Fig. 1G, lanes 6-8), but Brat-NHL still bound this fragment when the BoxB motif was mutated (hb 55-81 mut BoxB) (Fig. 1G, lanes 10-12). Similarly, Brat-NHL still bound the long hb RNA with high affinity when both BoxB sites were mutated (Fig. 2B, lanes 5,6), but mutation of both BoxA sites greatly impaired Brat-NHL binding (Fig. 2B, lanes 8,9), indicating that the Brat-NHL domain binds to the BoxA motif of the hb NREs. Notably, while mutating both BoxB sites did not affect the affinity of Brat-NHL for the hb RNA, it did abolish the appearance of the second, less well-defined Brat-RNA complex that appeared at high protein concentrations (Fig. 2B, marked by an asterisk).

To complement our binding studies, we also performed band shift assays with the purified RNA-binding domain of Pum (amino acids 1093-1426, Pum homology domain [Pum-HD]) (Supplemental Fig. 3; Zamore et al. 1997). As shown previously, the Pum-HD binds to hb mRNA with high affinity. Under our assay conditions, half of the free RNA was shifted into a protein-RNA complex at a concentration of $\sim 20 \mathrm{nM}$ Pum-HD (Supplemental Fig. 3B). In contrast to Brat-NHL and as shown previously (Zamore et al. 1997), binding of the Pum-HD to hb RNA was abrogated by mutation of both BoxB sites but not by mutation of both BoxA sites (Supplemental Fig. 3C, lanes 5,6 and 8,9, respectively). Thus, our data clearly indicate that the BoxB motif of the NRE recruits Pum, and the BoxA motif is responsible for Brat binding.

\section{Two Brat-binding sites are necessary for high-affinity binding}

The Pum-HD binds independently and with equal affinity to each of the two NREs, and no cooperative binding of two Pum-HD molecules occupying adjacent NREs was observed (Zamore et al. 1999). Consistently, mutation of either NRE1 $1_{\text {BoxB }}$ or NRE2 $2_{\text {BoxB }}$ did not abolish binding of the PUM-HD to hb RNA but simply eliminated the possibility of recruiting two PUM-HD molecules onto one hb RNA (Supplemental Fig. 3D).

In stark contrast, mutation of either NRE1 $1_{\text {BoxA }}$ or NRE2 $2_{\text {BoxA }}$ greatly impaired detectable binding of BratNHL to the hb RNA (Fig. 2C, lanes 8,9 and 11,12, respectively), indicating that two intact binding sites are necessary for the recruitment of the Brat-NHL domain in vitro. The requirement for two adjacent Bratbinding sites on one RNA molecule might explain our 


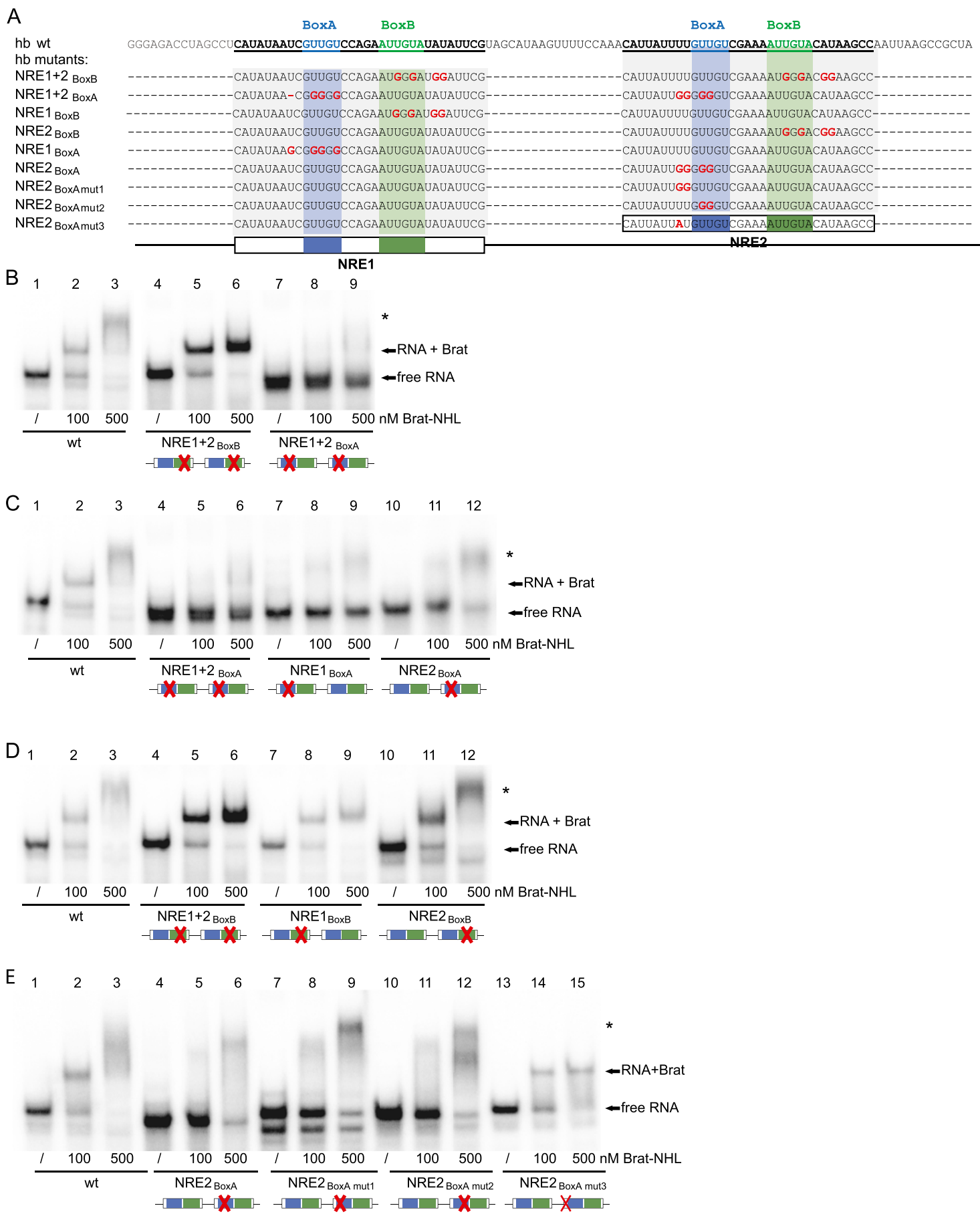

Figure 2. Mutations in BoxA, but not BoxB, abrogate binding of Brat-NHL to hb RNA. (A) Sequence of the hb RNA and its mutants used in binding assays. Mutated nucleotides are indicated by red letters. $(B-E)$ Recombinant Brat-NHL was incubated with ${ }^{32} \mathrm{P}-\mathrm{labeled}$ hb RNA or mutant RNAs as indicated and analyzed by native gel electrophoresis. Mutation of either BoxA site is sufficient to greatly impair RNA binding of Brat-NHL. Free RNA or RNA-protein complexes are indicated by black arrows. Asterisk $\left({ }^{\star}\right)$ denotes a less welldefined Brat-NHL-hb RNA complex appearing at high protein concentrations.

observation that the shorter $\mathrm{hb}$ fragments (hb $1-27$ and hb 55-81) that contain only one Brat binding site show a much lower affinity to Brat-NHL (Fig. 1D) compared with the long hb RNA containing two sites.

To complement our binding studies and further narrow down the Brat-binding motif, we also tested mutations in
NRE1 $1_{\text {BoxB }}$ or NRE2 $2_{\text {BoxB }}$ individually (Fig. 2D) as well as additional mutations in NRE2 $2_{\text {BoxA }}$ (Fig. 2E) on Brat-NHL binding. The two NRE2 $2_{\text {BoxA }}$ mutants (NRE2 $2_{\text {BoxA mut } 1}$ and

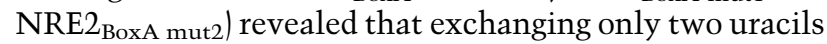
within or preceding the BoxA motif of NRE2 suffice to abrogate Brat-NHL binding to the hb RNA in vitro (Fig. 2E, 
lanes 8,9 and 11,12 , respectively), while mutation of NRE1 $1_{\text {BoxB }}$ (Fig. 2D, lanes 8,9 ) or the exchange of a uracil preceding the BoxA motif in NRE2 into an adenine

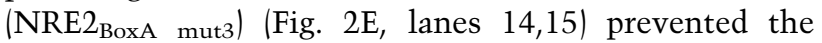
appearance of the less well-defined second Brat-NHL-hb RNA complex, an observation that we cannot currently explain but that might indicate a potential contribution of RNA folding to the accessibility of the Brat-binding sites.

\section{Stoichiometry of the Brat-NHL-hb RNA complex}

Although we observed only one high-affinity Brat-NHLhb RNA complex in our band shift assays (Fig. 1C) and the binding curves determined by MST did not indicate cooperative or independent binding of two Brat-NHL molecules (Fig. 1E), the presence of two adjacent Bratbinding sites and their requirement for high-affinity binding (Fig. 2C) suggested that two Brat-NHL moieties (possibly as a dimer) might bind to one hb RNA. To test this, static light scattering was used to determine the molecular weight of fractions eluted from an analytical gel filtration column. As shown in Supplemental Figure 4, only peaks corresponding to unbound Brat-NHL and a Brat-NHL:hb RNA 1:1 complex could be detected even when Brat-NHL was added at a 5.3-fold excess over hb RNA. Thus, in vitro, only one Brat-NHL domain binds to the hb RNA containing two binding sites.

\section{Pum-HD enhances Brat-NHL binding to the hb RNA}

An RNA-dependent interaction between Pum and Brat had previously been observed in yeast four-hybrid and in vitro pull-down assays (Sonoda and Wharton 2001), and gel filtration experiments performed by us similarly indicated that Brat-NHL and the Pum-HD formed a stable complex only in the presence of RNA (Supplemental Fig. 5). Since the NHL domain was described as a putative protein-protein interaction domain (Slack and Ruvkun 1998), these findings had previously been interpreted as a direct protein-protein interaction between RNA-bound Pum and Brat (Sonoda and Wharton 2001; Edwards et al. 2003). Given the close proximity of the BoxA and BoxB motifs, we asked whether the Pum-HD and Brat-NHL might influence each other's binding to the hb RNA.

To answer this question, we performed band shift assays with increasing amounts of Brat-NHL and either free hb RNA (Fig. 3A, lanes 1-7) or hb RNA that was prebound to the Pum-HD (Fig. 3A, lanes 8-14). While Brat-NHL bound the free RNA with a $K_{d}$ of $\sim 100 \mathrm{nM}$ (Figs. 1C, 3A; Supplemental Fig. 2B), a concentration of only $10 \mathrm{nM}$ Brat-NHL was sufficient to shift half of the Pum-HD-bound hb RNA into a slower-migrating complex containing Brat-NHL, the Pum-HD, and hb RNA (Fig. 3A, lane 11; Supplemental Fig. 3). At a concentration of $50 \mathrm{nM}$ Brat-NHL, all of the Pum-HD-bound RNA was shifted into the slower-migrating complex (Fig. 3A, lane 12). Thus, Brat-NHL binds to a Pum-HD-hb RNA complex with higher affinity than to free RNA, explaining previous findings (Edwards et al. 2003). The presence of the Pum-HD, however, could not overcome the requirement for two Brat-binding sites, as no detectable binding of the Brat-NHL domain to hb RNA occurred when the BoxA motif of either NRE2 (Fig. 3B) or of NRE1 (data not shown) was mutated even if the RNA was prebound to the Pum-HD. We also performed the reciprocal experiment, incubating increasing amounts of the Pum-HD with either free hb RNA or hb RNA that was prebound to Brat-NHL (Supplemental Fig. 6C). Correspondingly, the Pum-HD preferentially bound hb RNA that was prebound by Brat-NHL. Thus, binding of the Pum-HD or
A

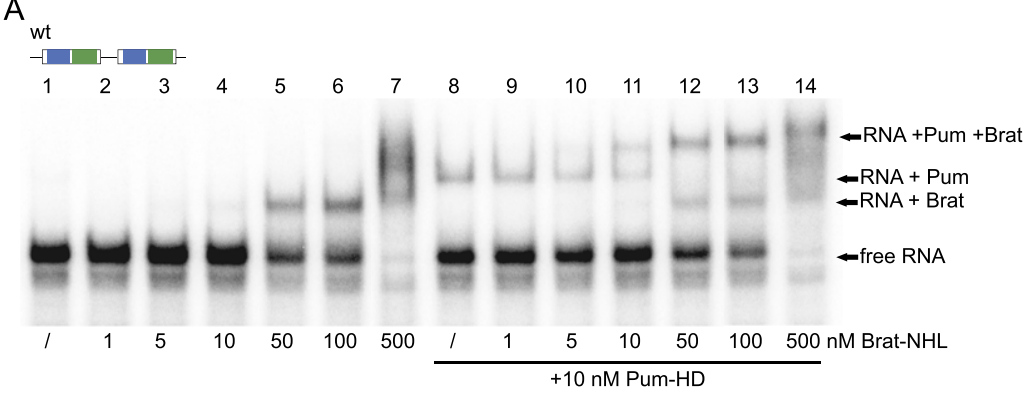

B

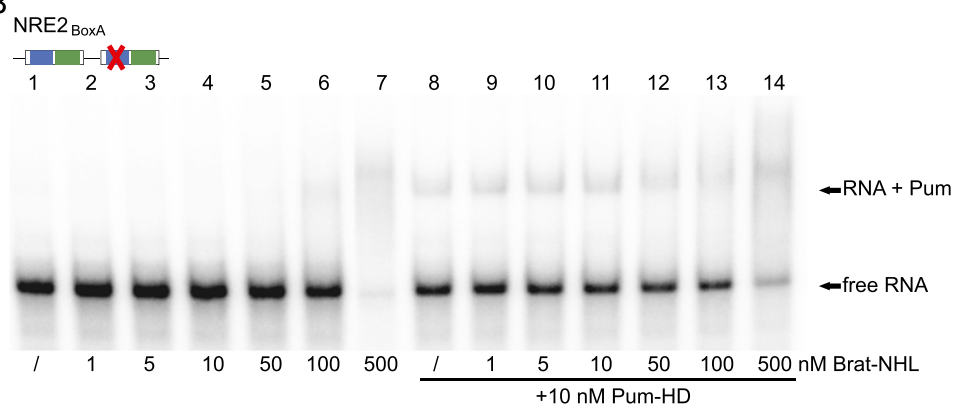

Figure 3. Binding of the Pum-HD to hb RNA facilitates Brat-NHL binding but does not overcome the requirement for two Brat-binding sites. (A) Binding of the Pum-HD and Brat-NHL to the hb RNA is not mutually exclusive, and preincubation of hb RNA with the Pum-HD facilitates Brat-NHL binding. Indicated amounts of Brat-NHL were mixed with ${ }^{32} \mathrm{P}$ labeled hb RNA alone (lanes 1-7) or hb RNA preincubated with $10 \mathrm{nM}$ Pum-HD (lanes 8-14). Complexes were separated by native gel electrophoresis. $(B)$ Binding of the Pum-HD to hb RNA does not overcome the requirement for two Brat-binding sites. Experiment was done as described in $A$ except that the hb mutant NRE2 $2_{\text {BoxA }}$ was used as a substrate for complex formation. 
Brat-NHL to RNA facilitates the recruitment of the other, an observation that could be explained by a stabilizing effect due to weak protein-protein interactions between the Pum-HD and Brat-NHL or by an altered, more accessible RNA structure that would facilitate binding of the second protein. It is currently unknown whether a stronger protein-protein interaction between Brat and Pum might occur in the context of the full-length proteins.

\section{Repression of hb by Brat is independent of Pum}

To test whether our in vitro binding studies have implications for the repression of the hb mRNA by Brat and Pum in vivo, we performed reporter gene assays in Dmel2 cells, a cell line derived from Drosophila embryonic Schneider cells (Fig. 4). The 100-nt hb 3' UTR fragment used above or respective mutants were fused to the coding sequence of firefly luciferase (FL) (Fig. 4A) and coexpressed with either full-length HA-tagged Pum or HA-tagged Brat and a Renilla luciferase (RL)-containing control vector.

Expression of HA-Brat or HA-Pum, but not of HAGawky (HA-GW) (a translational repressor not implicated in the regulation of $\mathrm{hb}$ ), led to a dose-dependent repression of the hb reporter (Fig. 4B; Supplemental Fig. 7B). The hb NRE1+2 $2_{\text {BoxA }}$ mutant, which did not bind Brat-NHL in our in vitro binding assays, was not repressed by HA-Brat, and the hb NRE1 $+2_{\text {BoxB }}$ mutant, which failed to recruit the Pum-HD (Fig. 4B), showed no repression by HA-Pum, clearly demonstrating that the BoxA motif is responsible for recruitment of and repression by Brat, while the BoxB motif recruits and confers repression by Pum. Additionally, these experiments demonstrate that repression by Brat and Pum can occur independently of each other, as the mutant that did not recruit Pum (NRE1+2 $2_{\text {BoxB }}$ ) was still repressed by Brat, and the mutant that did not bind Brat (NRE1+2 $2_{\text {BoxA }}$ ) was still repressed by Pum.

Contrary to our in vitro binding studies, mutation of each BoxA motif individually did not fully abrogate repression by Brat: Mutation of NRE2 $2_{\text {BoxA }}$ impaired repression by Brat, but mutation of NRE1 $1_{\text {BoxA }}$ had no appreciable effect on Brat-mediated repression, suggesting that one Brat-binding site is sufficient for Brat recruitment in vivo. These data also suggest that Brat exhibits lower affinity toward the BoxA motif of NRE1 than to that of NRE2, which is in agreement with our in vitro binding studies (Fig. 1D; data not shown).

Since Dmel2 cells express endogenous Brat and Pum (Cherbas et al. 2011; Harris et al. 2011; Weidmann and Goldstrohm 2012), we also performed knockdown experiments. As we lacked an antibody against endogenous Pum, we confirmed knockdown efficiency by the downregulation of coexpressed HA-tagged Pum (Fig. 4C, lanes 1,2). Knockdown of Pum relieved repression of the hb reporter (Fig. 4D), demonstrating its repression by endogenous Pum. Repression was dependent on two intact BoxB motifs, as the mutant hb reporter construct NRE1 $+2_{\text {BoxB }}$ was not affected or was only slightly affected by Pum knockdown. Notably, the NRE1+2 $2_{\text {BoxB }}$ hb re- porter generally showed elevated expression in all samples when compared with the other hb reporter constructs, indicative of repression relief due to mutation of the Pum-binding sites (see raw data in Supplemental Fig. 7A). For reasons that we cannot yet explain, knockdown of Brat did not relieve repression of any reporter construct tested (Fig. 4D), although Brat was expressed in Dmel 2 cells and was efficiently depleted by the knockdown approach (Fig. 4C, lanes 5,6), demonstrating a lack of repression by endogenous Brat in Dmel 2 cells. Possibly, the endogenous expression level of Brat in Dmel2 cells is not sufficient to repress the transfected reporter plasmids.

Under Pum knockdown conditions (Fig. 4E), the extent of Brat-mediated hb repression was comparable with that in untreated cells, again demonstrating that repression by Brat is independent of Pum.

To analyze other known Brat and Pum targets as well (Harris et al. 2011; Shi et al. 2013), we tested repression of myc and mad 3' UTRs by HA-Brat and HA-Pum overexpression (Fig. 4F). While the myc 3' UTR reporter was repressed $\sim 1.5$-fold by expression of HA-Brat or HA-Pum, repression of the mad 3' UTR reporter was observed only under HA-Brat but not HA-Pum overexpression conditions. Similarly, depletion of endogenous Pum did not cause repression relief of the mad 3' UTR reporter, while the expression of the myc and hb reporters was upregulated (Fig. 4G). Our data therefore suggest that mad might be a Pum-independent Brat target.

\section{The electropositive top surface of the NHL domain contacts RNA}

The six NHL repeats of the Brat-NHL domain fold into a six-bladed $\beta$ propeller (Fig. 5A,B; Edwards et al. 2003), a structure that serves as a platform for diverse molecular interactions (Stirnimann et al. 2010). Electrostatic calculations reveal an overall positive charge of the top surface (Fig. 5C; Edwards et al. 2003), and many positively charged and aromatic residues ideally suited for the interaction with RNA protrude from this surface. Strikingly, all mutations that cause a Brat mutant phenotype or that abrogate Brat function affect this surface (Arama et al. 2000; Sonoda and Wharton 2001; Harris et al. 2011). Additionally, this surface had been proposed to mediate the interaction between Brat and Pum (Sonoda and Wharton 2001; Edwards et al. 2003). Our finding of a direct RNA-binding activity of the Brat-NHL domain suggests that the described RNA-induced interaction between Brat and Pum (Sonoda and Wharton 2001; Edwards et al. 2003) might actually be an RNA-mediated interaction, and mutations affecting Pum binding might in fact be RNA-binding mutants.

To test this hypothesis, we performed band shift assays with recombinant Brat-NHL (Supplemental Fig. 1B) carrying point mutations on the top (reported to affect interaction with Pum) or the bottom (shown to have no effect on Pum binding) surface (Fig. 5D,E). While all top surface mutations greatly impaired (Y829A and R847A) (Fig. 5D, lanes 6,7 and 8,9, respectively) or completely abrogated (H802L and R875A) (Fig. 5D, lanes 4,5 and 


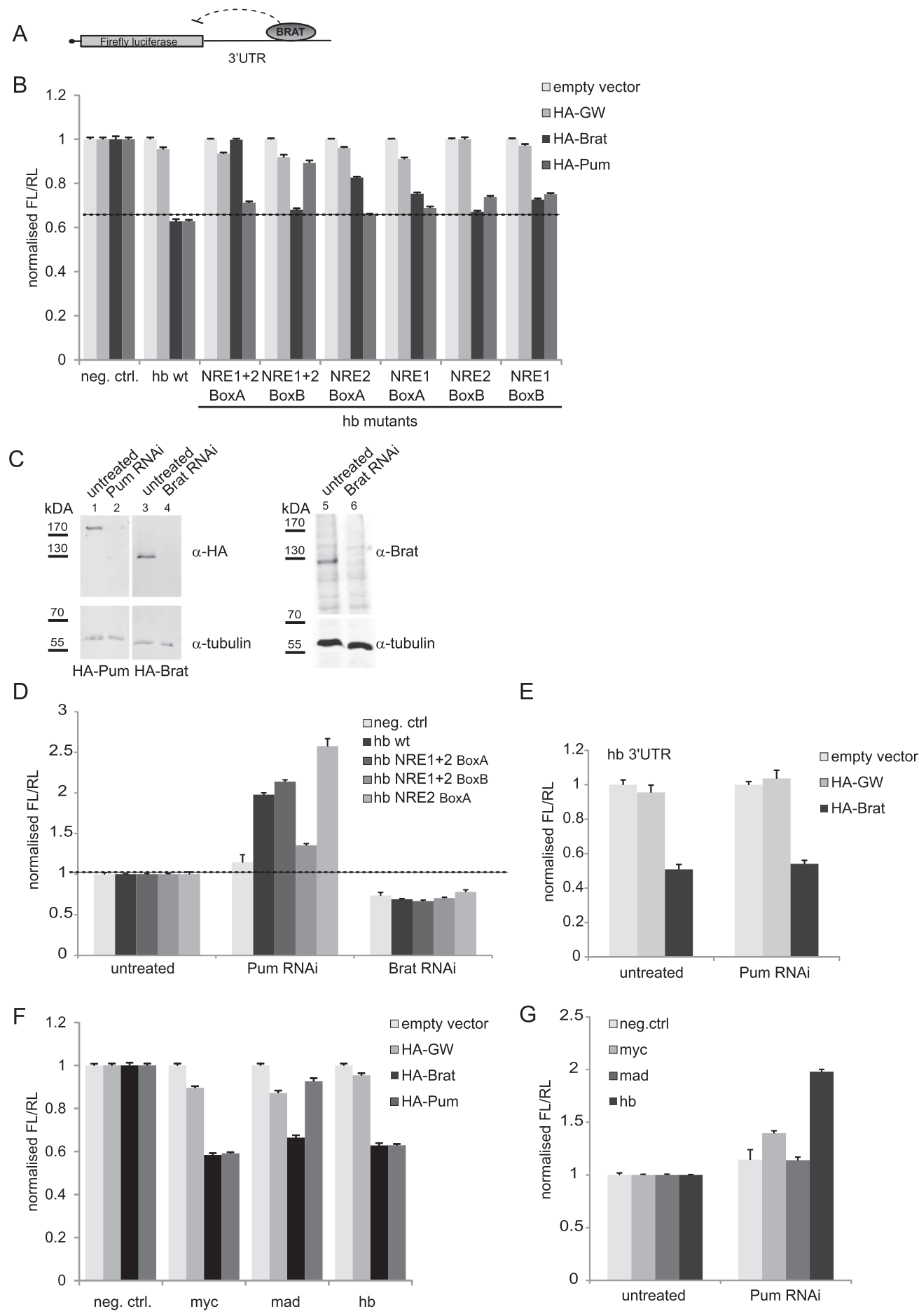

Figure 4. Repression by Brat is independent of Pum. (A) Schematic representation of the FL reporter constructs used in this study. The reporter constructs contain the FL coding sequence fused to a $3^{\prime}$ UTR of interest. In $B$ and $D-G$, the 100-nt hb 3' UTR fragment and its various mutants (as depicted in Fig. 2A) were analyzed. In $F$ and $G$, the $3^{\prime}$ UTRs of myc and mad were studied. (B) Dmel2 cells were cotransfected with plasmids expressing the indicated FL 3' UTR reporter constructs, a RL control, and the indicated HA fusion proteins. HA-GW, not known to regulate hb translation, served as an additional control. Values represent means of three independent experiments, each performed in triplicate, and error bars show standard error of mean. A representative experiment, including raw FL and RL values and normalization steps, is shown in Supplemental Figure 5. Expression of HA-Brat or HA-Pum, but not of HA-GW, led to an $\sim 1.5$-fold repression of the hb 3' UTR reporter. Mutations in BoxA abrogated repression by HA-Brat but not by HA-Pum, while mutations in BoxB impaired repression by HA-Pum but not by HA-Brat. $(C)$ Efficacy of dsRNA treatment was assayed by Western blotting. Due to the lack of an antibody against endogenous Pum, Pum depletion was assayed by cotransfected HA-Pum. $(D)$ Knockdown of endogenous Pum but not Brat relieves hb repression. Dmel2 cells were treated with dsRNA to Pum and Brat as detailed in the Supplemental Material. The indicated FL reporter constructs were cotransfected with a RL control vector, and reporter gene expression was analyzed as described in $B$. (E) Knockdown of endogenous Pum does not affect repression by Brat. Reporter gene assay was performed as described in $D$ except that plasmids expressing the indicated HA fusion proteins were cotransfected along with the luciferase constructs. $(F)$ Repression of myc and mad 3' UTRs by HA-Pum and HA-Brat. Experiment was performed as described in B. Expression of Brat leads to repression of both the myc and the mad 3' UTR reporter, while expression of Pum results in repression of myc but not of mad. (G) Knockdown of endogenous Pum relieves repression of hb and myc but not of mad. Experiment was performed as described in $D$. 
A
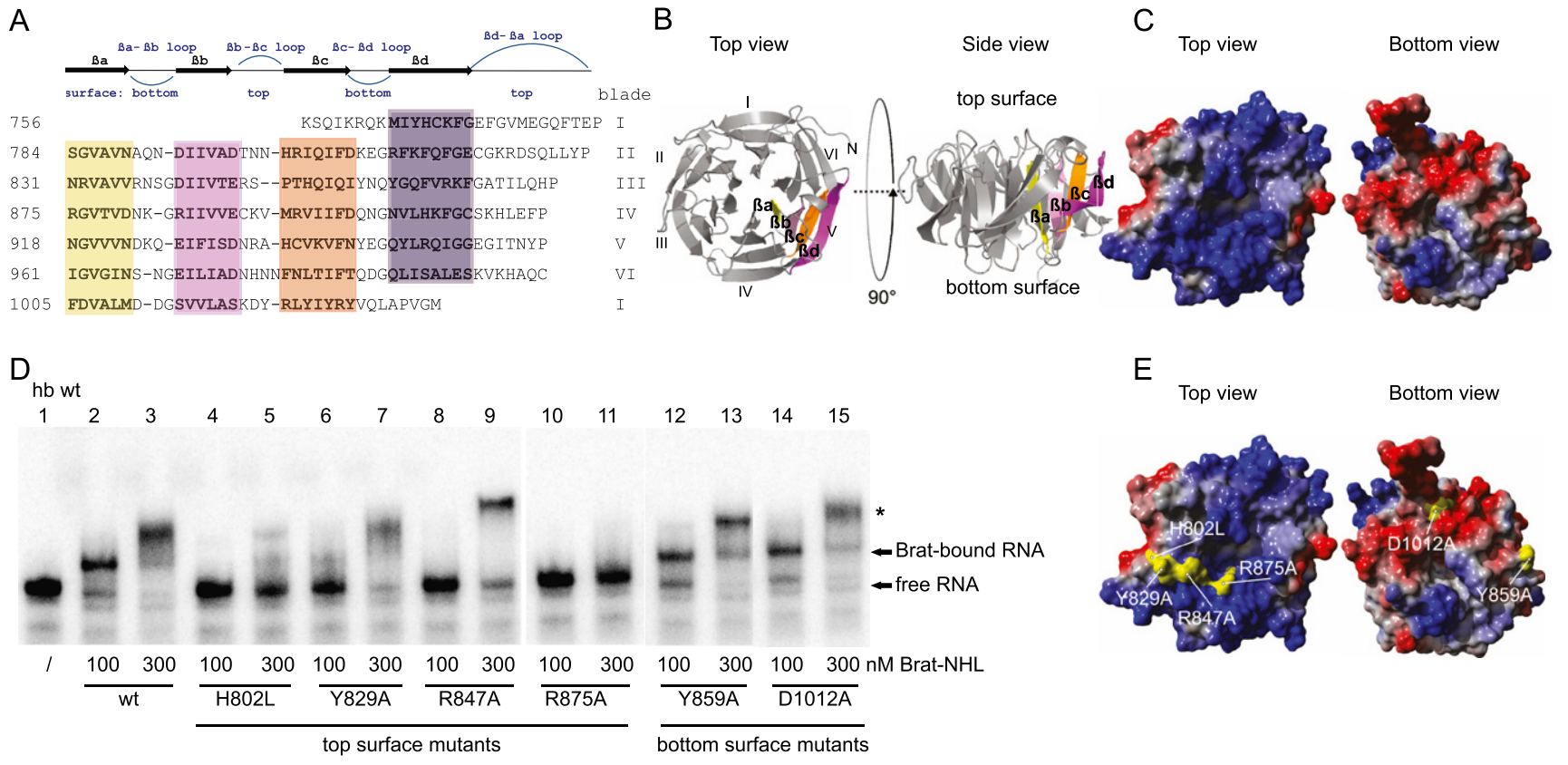

$\mathrm{E}$

Top view Bottom view

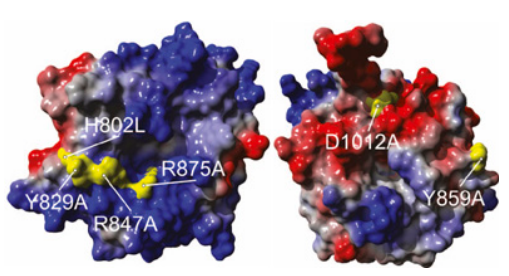

$\mathrm{F}$

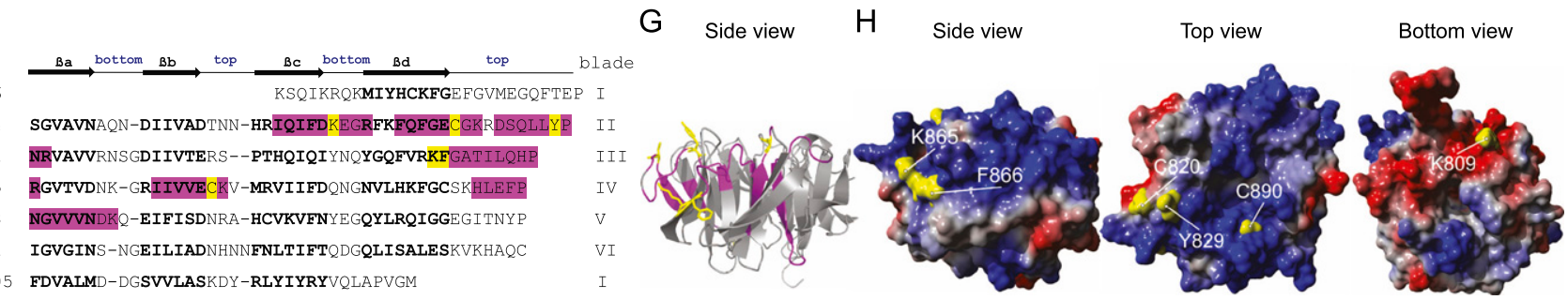

Figure 5. Residues on the top, electropositive surface of the NHL domain contact RNA. (A) Structure-based sequence alignment of the six NHL repeats that form the Brat-NHL domain (based on the crystal structure of Brat [PDB ID 1Q7F]) (Edwards et al. 2003). Secondary structure elements are depicted above the alignment. Residues that make up the $\beta$ strands are shown in bold and accentuated in yellow, pink, orange, and purple for $\beta$ strands $\beta a, \beta b, \beta c$, and $\beta d$, respectively. (B) Structure of the Brat-NHL domain looking from the top (left) or the side (right). Each blade of the six-bladed $\beta$ propeller is composed of four anti-parallel $\beta$ strands (termed $\beta$ a to $\beta d$ ) that are connected by flexible loop regions. By definition, the loops that connect $\beta \mathrm{b}$ with $\beta \mathrm{c}$ and $\beta \mathrm{d}$ with $\beta \mathrm{a}$ form the top surface of the molecule, while loops connecting $\beta$ a with $\beta \mathrm{b}$ and $\beta \mathrm{c}$ with $\beta \mathrm{d}$ make up the bottom surface. $\beta$ Strands of blade $\mathrm{V}$ are colored according to the sequence alignment shown in $A .(C)$ Electrostatic calculations reveal an electropositive top surface and an electronegative bottom surface. Negative surface potential is shown in red, and positive surface potential is shown in blue. $(D)$ Mutation of the top surface residues greatly impairs BRAT-NHL RNA binding. Recombinant Brat-NHL or the indicated point mutants were incubated with ${ }^{32}$ P-labeled hb RNA and analyzed by native gel electrophoresis. $(E)$ Electrostatic surface potential, with the residues tested in $D$ indicated in yellow. $(F-H)$ Summary of in vitro cross-linking experiments. Brat-NHL-hb RNA complexes were UV-cross-linked, and, following isolation, peptide-oligonucleotide cross-links were analyzed by liquid chromatography (LC)/MS. Identified peptides are shown in purple, while residues sequenced as RNA adducts are highlighted in yellow. Five out of six peptides span the top surface.

10,11 , respectively) binding of the Brat-NHL domain to hb RNA, mutations on the bottom surface had no effect. A more thorough titration (Supplemental Fig. 8) confirmed that the R875A mutant completely lost RNA binding, while the R874A mutant lost high-affinity binding but still showed the appearance of the second, less well-defined band at high protein concentrations (Supplemental Fig. 8, marked by an asterisk). MST experiments confirmed the lack of RNA binding for the R875A mutant (data not shown).

In addition, we also took an unbiased approach using UV-cross-linking followed by MS to identify proteinRNA contact sites (Luo et al. 2008; Kramer et al. 2011).
In vitro formed Brat-NHL-hb RNA complexes were UVcross-linked, and, following their enrichment by titaniumdioxid chromatography, peptide-oligonucleotide cross-links were analyzed by liquid chromatography (LC)/MS. As summarized in Figure 5, F-H, five out of six peptides that were identified (highlighted in pink) span the top surface of the NHL domain. Within these peptides, six residues (Fig. 5F-H, highlighted in yellow) were identified as nucleotide adducts, indicating their direct contact with or very close proximity to RNA. Three of these residues (including Y829, whose mutation lead to greatly impaired hb RNA binding in our in vitro binding assay) (Fig. 5D) are located on the top surface, while two 
others (K865 and F866) lie in a positively charged patch at the circumference (Fig. $5 \mathrm{H}$ ).

\section{Individual mutation of top surface residues abrogates Brat-mediated repression}

Next, we tested the effect of single NHL domain point mutations on Brat-mediated repression using the previously introduced luciferase reporter assay. Here, we also included residues that we identified in our MS approach (Fig. 6). While repression was unaffected by mutation of residues that are located on the bottom surface of the NHL domain, almost all top surface mutations (except C820A and Y829A) impaired or abrogated Brat-mediated repression (Fig. 6A, top). Repression relief was due to a defect in RNA binding, as the same mutations showed no effect on Brat-mediated repression when the protein was artificially tethered to the RNA (Fig. 6B). As summarized in Figure 6, C-E, eight out of nine top surface mutants tested either compromised Bratmediated reporter gene repression (Fig. 6A) or impaired Brat-NHL-hb RNA binding in in vitro binding assays (Fig. $5 \mathrm{D}$ ), while none of the bottom surface mutants showed an effect in either of the two assays, clearly demonstrating that the top surface of the Brat-NHL domain is the RNAbinding platform. Notably, the bottom surface mutant R837D, which was reported to disrupt the interaction to the cap-binding protein 4EHP (Cho et al. 2006), showed no effect in our reporter assays, possibly due to the limitations of this assay.

\section{A positively charged top surface distinguishes the NHL domain of TRIM-NHL proteins from other NHL domain-containing proteins}

The NHL repeat sequence (Fig. 5A) is found in a variety of different proteins from eukaryotic as well as prokaryotic organisms (Slack and Ruvkun 1998), with the number of readily identifiable repeats varying between two and six. In addition to Brat, the crystal structures of the six-bladed NHL domains of the receptor serine/threonine protein kinase PknD of Mycobacterium tuberculosis (Good et al. $2004)$ and of the peptidyl- $\alpha$-hydroxyglycine- $\alpha$-amidating lyase (PAL) of Rattus norvegicus (Chufan et al. 2009) have been solved. While the NHL domain of PknD serves as an extracellular sensor domain for a so far unidentified ligand (Good et al. 2004), the NHL domain of PAL catalyzes the second and last steps in the amidation of neuropetides, and its top surface harbors the active site residues (Chufan et al. 2009). Evidently, different NHL domains have evolved to accommodate very distinct binding partners. Calculation of the electrostatic surface potential revealed that, in contrast to the overall positively charged top surface of the RNA-binding NHL domain of Brat, the top surface of the NHL domains of PknD and PAL show an overall negative surface charge (Fig. 7).

To gain more insight, we performed structural homology modeling for several six-bladed NHL domains, including all human, fly, and worm TRIM-NHL proteins, and calculated their electrostatic surface potential. As shown in Figure 7, the NHL domains of all TRIM-NHL proteins show largely positively charged top surface areas, indicative of their potential to bind negatively charged molecules such as nucleic acids. In agreement with recent large-scale mRNA interactome studies (Baltz et al. 2012; Castello et al. 2012; Kwon et al. 2013), the TRIM-NHL proteins may therefore be direct RNA-binding proteins, and RNA binding is likely to be mediated by their NHL domains.

\section{Discussion}

In this study, we identify the NHL domain of Drosophila Brat as a novel, sequence-specific ssRNA-binding domain. We show that the positively charged top surface of the NHL domain contacts RNA, and mutations of single residues on this surface abrogate Brat-NHL binding to the hb mRNA in vitro and impair Brat-mediated translational repression in vivo. Notably, all mutations known to date that cause a Brat mutant phenotype affect the NHL domain. The strongest alleles are NHL domain truncations, while weaker alleles carry point mutations of top surface residues (Arama et al. 2000; Sonoda and Wharton 2001), strongly suggesting that RNA binding is key to Brat function.

The RNA-dependent interaction between the BratNHL domain and the RNA-binding domain of Pum, which had been observed in yeast four-hybrid assays and in vitro binding studies (Supplemental Fig. 6; Sonoda and Wharton 2001; Edwards et al. 2003), had previously been interpreted as a protein-protein interaction between RNA-bound Pum and Brat (Sonoda and Wharton 2001; Edwards et al. 2003). RNA requirement for the interaction was explained by an assumed RNA-induced conformational change of the Pum-HD that would allow subsequent Brat binding (Sonoda and Wharton 2001; Edwards et al. 2003). Our data provide the basis for a modification of the current model of the $\mathrm{hb}$ mRNA regulation by the Pum-Nos-Brat complex: We show that Brat and Pum contact the RNA independently of each other, and, in addition, translational repression by Brat can occur independently of Pum (Fig. 6F). Residues previously thought to mediate the direct interaction between Brat and Pum (Edwards et al. 2003) are in fact important for RNA binding, indicating that the RNA-dependent interaction between Brat and Pum is RNA-mediated. While a complex of Pum, Nos, and Brat seems to be required for correct abdominal segmentation in Drosophila melanogaster, the dispensability of Pum for Brat's RNAbinding activity and Brat-mediated translational repression strongly suggests that Pum-independent Brat targets exist. Mad, which we found to be repressed by overexpression of Brat-but not by Pum-in Dmel2 cells, might be such a target.

Apart from its role during embryogenesis, Brat also functions in other developmental contexts; e.g., during the development of the larval brain. The differences between Brat and Pum mutant phenotypes might indicate Pum-independent Brat functions. Whether these functions require the RNA-binding activity of Brat or Brat cooperates with RNA-binding proteins other than Pum in these conditions needs to be determined. 
A

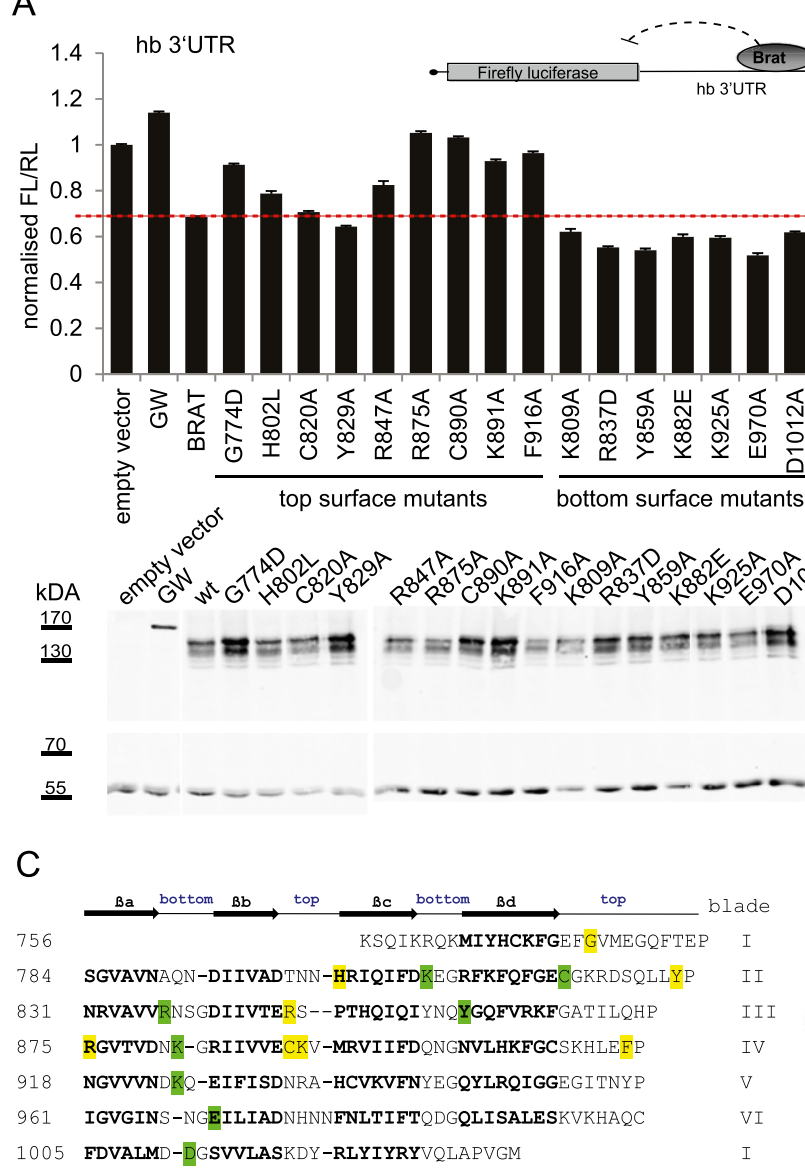

B

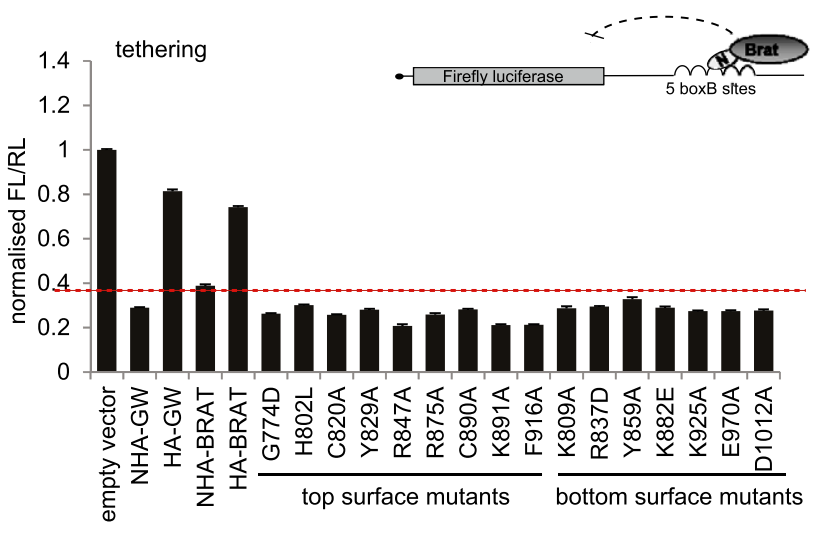

$\mathrm{F}$

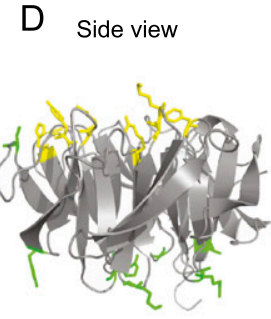

E
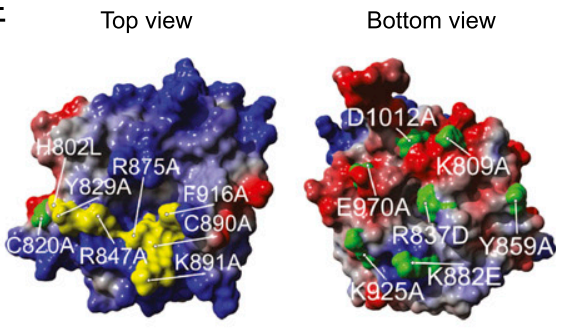

Brat-NHL

Pum-HD

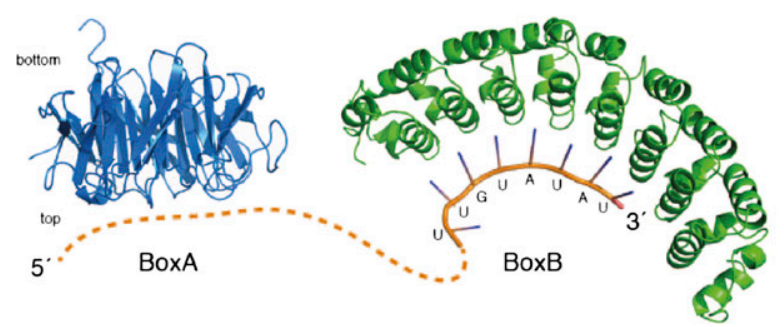

Figure 6. Mutation of the top surface residues abrogates Brat-mediated repression. $(A)$ Repression of the hb 3' UTR by HA-Brat or the indicated HA-Brat point mutants in Dmel2 cells. (Top) Dmel2 cells were cotransfected with plasmids expressing the FL hb 3' UTR reporter, the indicated HA fusion proteins, and a RL control plasmid. FL was normalized to RL, and values of normalized FL produced in the presence of an empty control vector were set to 1 . (Bottom) Protein expression was analyzed by Western blotting. $(B)$ Tethering experiment. Mutations that impair Brat-mediated hb repression have no effect when Brat is artificially tethered to the RNA via fusion to the $\lambda$ phage N-peptide (N), targeting the fusion protein to hairpin structures in the 3' UTR of the reporter (see the inset). Dmel2 cells were cotransfected with plasmids expressing FL-5boxB, the indicated NHA or HA fusion proteins, and a RL control plasmid. FL was normalized to RL, and values of normalized FL produced in the presence of an empty control vector were set to 1. Tethering of GW served as a positive control. Values represent means of three independent experiments, each performed in triplicate, and error bars show standard error of the mean. $(C, D)$ Summary of mutagenesis studies. Mutations that affect Brat RNA binding are shown in yellow, while mutations that have no effect on Brat RNA binding are depicted in green. All mutations that lie on the top surface of the molecule, except C820A, either impair Brat-mediated hb repression $(A)$ or in vitro binding of the Brat-NHL domain to hb RNA (Fig. 5D). (F) Model of the Brat:Pum:RNA repressor complex. The Brat-NHL and the Pum-HD contact the RNA directly. A ribbon representation of the Brat-NHL domain (Protein Data Bank [PDB] ID: 1Q7F, chain A) is shown colored in blue, and a ribbon representation of the Pum-HD (PDB ID: 3H3D, chain X) domain is shown colored in green. A ribbon representation of the NRE1 RNA is shown in orange and purple. Binding of the D. melanogaster Pum-HD to the NRE1 sequence was modeled by superposition of the domain on the structure of the Homo sapiens Pum-HD of Pumiliol bound to the NRE1 sequence (PDB ID: 1M8X, chain A) using the align algorithm implemented in Pymol. Protein domains and ribbon representations of the RNA are drawn to scale. The dotted orange line indicates the $5^{\prime}$ region of the NRE1 RNA containing BoxA and, in the absence of structural information, is not drawn to scale. 


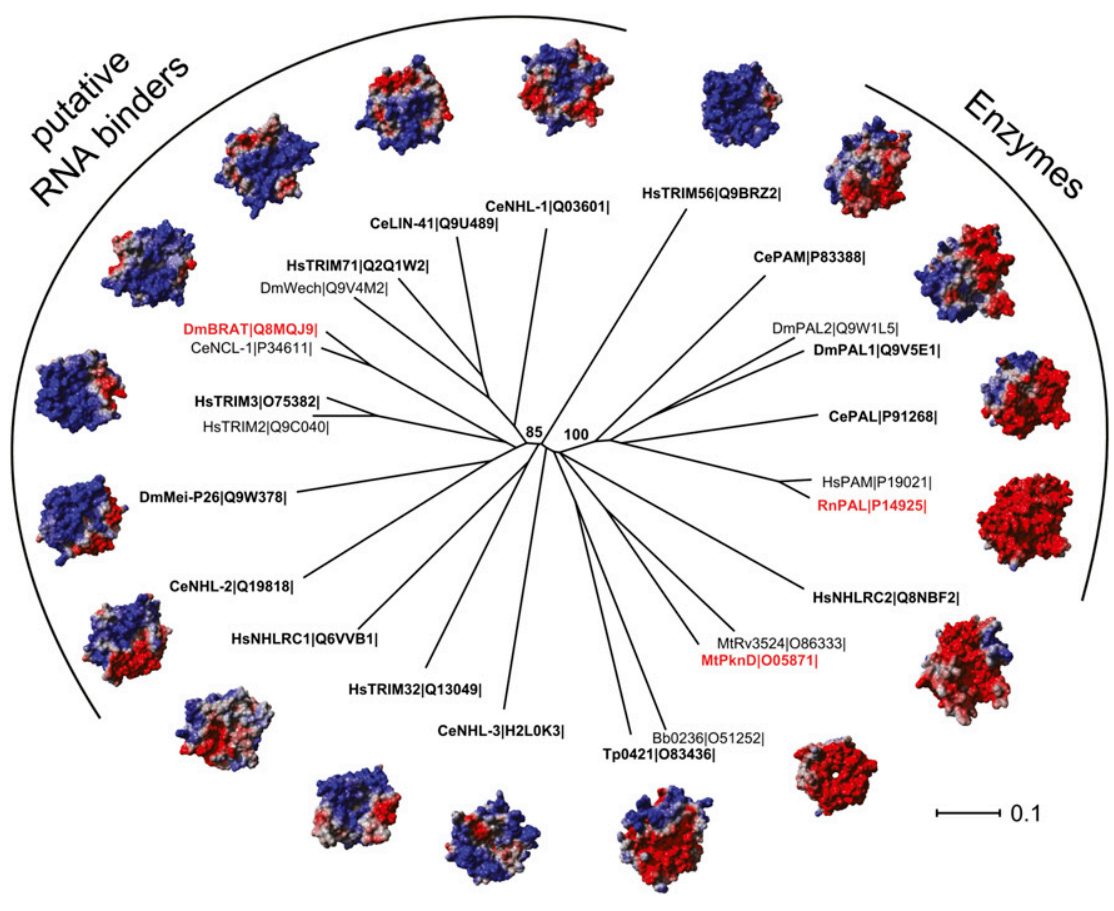

Figure 7. Phylogeny of six-bladed NHL domains. The neighbor-joining tree was derived from a structure-based multiple sequence alignment as described in the Materials and Methods. Characteristic proteins are represented by their known threedimensional structure (name in red) or by homology models (name in bold and black). The subfamilies of putative RNA binders and of enzymes are separated from the rest by a bootstrap value of $85 \%$ or $100 \%$, respectively. For each structure, Particle Mesh Ewald long-range electrostatic calculations were performed in YASARA and used to color-code the solvent-accessible surface: A negative charge is indicated by a red surface, and a positive charge is indicated by a blue surface. Abbreviations for protein names and species are given next to the Uniprot ID. (Bb) Borrelia burgdorferi; (Ce) Caenorhabditis elegans; (Dm) Drosophila melanogaster; (Hs) Homo sapiens; (Mt) Mycobacterium tuberculosis; (Rn) Rattus norvegicus; (Tp) Treponema pallidum. The length of the horizontal bar corresponds to 0.1 substitutions per site.
Pum proteins bind to the well-defined consensus sequence UGUA(N)AUA (Gerber et al. 2006). The bipartite NRE, composed of a BoxA and a BoxB motif, has been considered a specialized Pum-binding site, characterized so far only for Drosophila (Wang et al. 2002). However, only the BoxB motif, which conforms to the Pum consensus, binds Pum with high affinity (Zamore et al. 1997; Wang et al. 2002). Here we show that the BoxA motif is a high-affinity binding site for Brat. In addition to the $\mathrm{hb}$ 3' UTR, NREs are found in the 3' UTRs of bicoid and cyclin B (Wharton and Struhl 1991; Asaoka-Taguchi et al. 1999). It is tempting to speculate that NREs generally recruit Brat in addition to Pum. The close proximity of the Brat- and Pum-binding sites and the occurrence of the NREs in different 3' UTRs might indicate a close relationship between Brat and Pum. Our in vitro binding assays, carried out with the isolated RNA-binding domains, show that Brat and Pum facilitate each other's binding to the hb RNA. Counter to our expectations, we did not detect an additive or even cooperative effect of Brat and Pum in reporter gene assays (Supplemental Fig. 7B), possibly due to the limitations of this assay.

One prominent feature of Brat is its role as a growth suppressor and differentiation factor (Frank et al. 2002; Betschinger et al. 2006; Lee et al. 2006; Harris et al. 2011) that becomes most evident as a tumorous overproliferation of the larval brain in flies that lack functional Brat (Arama et al. 2000). During asymmetric neuroblast divisions, Brat is confined to the differentiating daughter cell, where it is needed to stop proliferation and promote differentiation (Betschinger et al. 2006; Lee et al. 2006). A critical Brat-interacting partner in this process is the asymmetrically segregating cell fate determinant Miranda (Mira) (Betschinger et al. 2006; Lee et al. 2006). Notably, the same residues that we identified as RNA contact sites have previously been shown to mediate the interaction between Brat and Mira (Lee et al. 2006). It will thus be interesting to test the RNA requirement of this interaction and identify the RNAs potentially involved.

The NHL domain folds into a six-bladed $\beta$ propeller, a structure that cursorily resembles that of WD40 domains (Edwards et al. 2003). Initially recognized as versatile protein-binding domains, recent evidence suggests that $\beta$-propeller structures also evolved as platforms for nucleic acid binding (Stirnimann et al. 2010). The recent crystal structure of the DNA damage-binding protein 1 (DDB1)/DDB2 heterodimer, a protein complex involved in DNA repair, revealed that the top surface of the seven-bladed WD40 domain of DDB2 directly binds to DNA in a sequence-unspecific manner (Scrima et al. 2008). Many basic residues are located on this surface and are either in direct contact with or line the path of the DNA phosphate backbone along the propeller. Another example is the WD40 domain of Gemin5 that sequencespecifically binds to snRNAs (Lau et al. 2009). In this case, the 13-repeat-containing WD40 domain is predicted to form a tandem $\beta$-propeller structure, and basic and aromatic residues of one of the propellers' top surfaces contact the RNA. Additionally, the WD40 domain was suggested as a potential new RNA-binding domain in recent mRNA interactome studies, which were carried out in HEK293, HeLa, and mouse embryonic stem (mES) cells (Baltz et al. 2012; Castello et al. 2012; Kwon et al. 2013). Using UV-cross-linking followed by MS, these studies identified direct RNA-binding proteins globally and extended the number of putative RNA-binding proteins, many of which do not harbor any classical RNAbinding motif. The 28 WD40 domain containing proteins that were identified in this study are characterized by an 
enrichment of positively charged and aromatic residues (Castello et al. 2012; Kwon et al. 2013).

To provide a global overview over the nucleic acidbinding potential of NHL domains, we used the electrostatic surface potential of structural homology models as well as solved structures as an indication of the probability of an NHL domain binding to RNA (Fig. 7). Strikingly, while the NHL domains of all TRIM-NHL proteins are predicted to contain a positively charged top surface or at least contain large, positively charged patches suitable for accommodating RNA, the top surfaces of other NHL domains-for example, those with known enzymatic activities (e.g., RnPAL) - display an overall negative charge.

The family of TRIM-NHL proteins comprises three members in flies, four members in Caenorhabditis elegans, and five members in mammals, and many of them have been linked to RNA metabolism. For example, several TRIM-NHL proteins (including Drosophila Brat, Mei-P26, and Wech/Dappled; C. elegans NHL-2 and LIN-41; or mammalian TRIM2, TRIM3, TRIM32, and TRIM71) were found associated with RNA-protein complexes (RNPs) (Kanai et al. 2004; Duchaine et al. 2006; Neumuller et al. 2008; Hammell et al. 2009; Rybak et al. 2009; Schwamborn et al. 2009; Chang et al. 2012; Li et al. 2012, 2013; Loedige et al. 2013), and, in some cases, these interactions were shown to be dependent on either the RNA (Hammell et al. 2009; Chang et al. 2012; Li et al. 2012) or the respective NHL domain within the RNP (Neumuller et al. 2008; Schwamborn et al. 2009; Chang et al. 2012; Loedige et al. 2013). In addition to Brat, MeiP26 and TRIM71 were also recently shown to repress translation (Chang et al. 2012; Li et al. 2012, 2013; Loedige et al. 2013), and C. elegans LIN-41 and human TRIM71/ LIN-41 regulate expression of the transcription factors LIN-29 and EGR1, respectively, possibly at the level of translation (Slack et al. 2000; Worringer et al. 2014). In all cases, repression depends on the NHL domain (Slack et al. 2000; Chang et al. 2012; Li et al. 2012; Loedige et al. 2013). In support of a direct RNA-binding activity, the aforementioned mRNA interactome studies identified TRIM71 and TRIM56 as putative novel RNA-binding proteins (Baltz et al. 2012; Castello et al. 2012; Kwon et al. 2013), and, in case of TRIM71, direct RNA-binding was verified and mapped to the NHL domain (Kwon et al. 2013). The cell type-specific expression of other TRIM-NHL proteins has presumably precluded their identification in these studies, as neither TRIM2, TRIM3, nor TRIM32 are expressed in HEK293, HeLa, or mES cells to significant amounts (Reymond et al. 2001; Baltz et al. 2012; Kwon et al. 2013).

Although RNA binding appears to be common to all TRIM-NHL proteins, their diverse biological roles suggest that they have distinct sets of RNA-binding partners. Our data suggest that this sequence-specific RNA binding is mediated by their NHL domains.

\section{Materials and methods}

\section{DNA constructs}

The coding sequence of full-length Brat, the Brat-NHL domain, and the 3' UTRs or 3' UTR fragments of hb, myc, and mad, were amplified from Drosophila embryonic lysate cDNA. The coding sequence of full-length Pum or the Pum-HD were amplified from the Drosophila Genomics Resource Center clone number SD07661.

The PUM-HD (for, CTCCGCGGTGGTTCTCGCCTTCTCG AAGATTTCCGC; rev, TTATTAGAATTCTTACTTCTCCAAC TTGGCATTGAT) and the Brat-NHL domain (for, CTCCG CGGTGGTAAGTCGCAGATCAAGCGACAGA; rev, CTACT AGTCGACTTACATACCCACTGGCGCCA) were cloned into the pHUE expression vector using SacII/EcoRI or SacII/SalI sites, respectively. The 101-nt-long fragment of the hb 3' UTR that contains both NREs was amplified with the following primers: for, TAATACGACTCACTATAGGGAGACCTAGCCTCATAT AATCGTTGTCCAGAATTGTATA; and rev, AGAATTAGCGG CTTAATTGGCTTA. The forward primer contains a T7 polymerase promoter sequence for subsequent in vitro transcription and an 11-nt-long adapter sequence that would allow pull-down of the RNA but was not used in this study. To permit sitedirected mutagenesis, the PCR product was cloned into the pGEMTeasy vector (Promega). Reporter plasmids pAC-NHAGW, pAC-HA-GW, and pAC-FL-5boxB were a kind gift of M. Chekulaeva and have been described (Chekulaeva et al. 2009). The pAC-RL control vector was kindly provided by J. Medenbach. NHA-Brat, HA-Brat, and HA-Pum were generated by replacing the GW insert in pAC-NHA-GW or pAC-HA-GW with the respective Brat or Pum coding sequence using SbfI/NotI or Sbfi/EcoRI sites, respectively. Full-length Brat was amplified with the following primers: for, ATATATCCTGCAGGCA TGGCGTCCTCACCGACACCATCTCTGGACTC; and rev, ATATATGCGGCCGCTTACATACCCACTGGCGCCAGTTG GACATAGC. Full-length Pum was amplified with the following primers: for, ATATATCCTGCAGGCATGAAGTTTTTGGGTG GTAACGATGATC; and rev, ATATATGAATTCAATTTGTTA TTTCCTTTACAGCACAACGTTG. The FL-3'UTR constructs were generated by replacing the 5boxB sequence from pAC-FL5boxB with myc (for, ATATATGCTAGCGCGCTCGGTTAGTG GATAGT; rev, ATATATCTCGAGTGTTTCGTTTCTCCGCT AGG), mad (for, ATATATGCTAGCCCTCAATGGAGACGGAA GAG; rev, ATATATCTCGAGAAGGCAATTTTCTCGTGGTC), or $\mathrm{hb}$ (for, ATATATGCTAGCCATATAATCGTTGTCCAGAA; rev, ATATATCTCGAGAGAATTAGCGGCTTAATTGG) 3' UTRs or 3' UTR fragments using NheI/Xho sites. Point mutations were introduced into pGEMT-hb or pHUE-BRAT-NHL by site-directed mutagenesis (Zheng et al. 2004) with the primers listed in Supplemental Table 2. In the case of full-length Brat, all point mutations were first introduced into pHUE-Brat-NHL and further subcloned into NHA-Brat or HA-Brat using EcoRI/NotI sites. The correctness of all plasmids was verified by sequencing.

\section{RNA}

Small RNAs ( $\leq 27$ base pairs [bp]) were ordered chemically synthesized (Biomers). The long hb 3' UTR fragment and its point mutants were in vitro transcribed from $2 \mu \mathrm{g} / \mathrm{mL}$ PCRamplified DNA templates using $0.1 \mathrm{mg} / \mathrm{mL} \mathrm{T7}$ polymerase in 30 $\mathrm{mM}$ Tris (pH 8), $25 \mathrm{mM} \mathrm{MgCl}_{2}, 0.01 \%$ Triton-X100, 1 mM DTT, $10 \mathrm{mM}$ each NTP, $2 \mathrm{U} / \mathrm{mL}$ pyrophosphatase (New England Biolabs), and $2 \mathrm{mM}$ spermidin for $4 \mathrm{~h}$ at $37^{\circ} \mathrm{C}$. In vitro transcribed RNA was gel-purified on a $15 \%$ polyacrylamide gel containing 7.5 M urea (SequaGel systems, National Diagnostics).

\section{Protein expression and purification}

All purification steps were performed at $4^{\circ} \mathrm{C}$, and protein concentration was determined spectrophotometrically at 280 $\mathrm{nm}$. Proteins were produced as $\mathrm{His}_{6}$-ubiquitin fusion using the 
pHUE vector system as described previously (Catanzariti et al. 2004; Baker et al. 2005).

Drosophila Brat-NHL and Pum-HD were expressed in Escherichia coli BL21(DE3). Cells were grown at $37^{\circ} \mathrm{C}$ to an $\mathrm{OD}_{600 \mathrm{~nm}}$ of 0.6 , and protein expression was induced by the addition of $1 \mathrm{mM}$ IPTG overnight at $23^{\circ} \mathrm{C}$.

Cells were lysed by incubation in HisA buffer $150 \mathrm{mM}$ Tris at $\mathrm{pH} 8,1 \mathrm{M} \mathrm{NaCl}, 5 \%$ glycerol, $10 \mathrm{mM}$ imidazol) containing $1 \mathrm{mg} / \mathrm{mL}$ lysozyme, $1 \mathrm{mM}$ AEBSF, and $5 \mathrm{U} / \mathrm{mL}$ benzonase and subsequent sonication. The supernatant obtained by centrifugation (48.000 $\mathrm{g}$ for $40 \mathrm{~min}$ at $4^{\circ} \mathrm{C}$ ) was loaded on a HiTrap IMAC FF 5 -mL column charged with $\mathrm{Ni}^{2+}$ and eluted by buffer HisB $(50$ $\mathrm{mM}$ Tris at $\mathrm{pH} 8,1 \mathrm{M} \mathrm{NaCl}, 200 \mathrm{mM}$ imidazol). His 6 -ubiquitin fusion protein-containing fractions were pooled, and the His6ubiquitin moiety was cleaved off by incubation with the Usp2cc enzyme overnight at $4^{\circ} \mathrm{C}$ in buffer HisB containing $1 \mathrm{mM}$ DTT. The protein solution was subsequently applied to a HiPrep Superdex 75 26/60 column equilibrated in $20 \mathrm{mM}$ Tris ( $\mathrm{pH} 8$ ), $150 \mathrm{mM} \mathrm{NaCl}$, and $1 \mathrm{mM} \mathrm{DTT}$. Fractions containing highly pure protein were pooled, glycerol was added to $5 \%$, and samples were flash-frozen in liquid nitrogen and stored at $-80^{\circ} \mathrm{C}$.

\section{${ }^{32} P$ labeling of RNA}

In vitro transcribed RNA ( 30 pmol) was dephosphorylated by 0.1 U/ $\mu$ L FASTAP (Fermentas) in $1 \times$ PNK A buffer (Fermentas) for $30 \mathrm{~min}$ at $37^{\circ} \mathrm{C}$ before FASTAP was heat-inactivated for $20 \mathrm{~min}$ at $75^{\circ} \mathrm{C}$. Subsequently, $30 \mu \mathrm{Ci} \gamma^{32} \mathrm{P}$-ATP (Hartmann Analytic) and T4 PNK (final concentration $0.5 \mathrm{U} / \mu \mathrm{L}$; Fermentas) were added, and the $5^{\prime}$ phosphorylation reaction was carried out in $1 \times$ PNK A buffer for $30 \mathrm{~min}$ at $37^{\circ} \mathrm{C}$. T4-PNK was heat-inactivated for $10 \mathrm{~min}$ at $75^{\circ} \mathrm{C}$. Free $\gamma^{32} \mathrm{P}$-ATP was removed by desalting on a G25 spin column (GE Healthcare) according to the manufacturer's instructions. $5^{\prime}{ }^{32} \mathrm{P}$-labeled RNA was stored at $-20^{\circ} \mathrm{C}$ until further use. Chemically synthesized small RNAs (30 pmol; Biomers) were directly phosphorylated by T4-PNK following the same protocol.

\section{EMSA}

Radioactively labeled RNA ( $~ 500 \mathrm{pM}$ to $2 \mathrm{nM})$ was incubated at $4^{\circ} \mathrm{C}$ with the indicated protein concentrations in buffer $10 \mathrm{mM}$ MOPS, $50 \mathrm{mM} \mathrm{KCl}, 5 \mathrm{mM} \mathrm{MgCl} 2,30 \mu \mathrm{g} / \mathrm{mL}$ heparin, $5 \%$ glycerol, $1 \mathrm{mM}$ DTT) containing a 1000 -fold excess $(\sim 2 \mu \mathrm{M})$ of unlabeled yeast tRNA (Ambion) for $30 \mathrm{~min}$. Complexes were resolved on $6 \%$ native polyacrylamide gels /acrylamid/bisacrylamid 37.5:1; Serva) containing 5\% glycerol and $0.5 \times$ TB buffer (45 mM Tris, $45 \mathrm{mM}$ borate). Electrophoresis was carried out for $3 \mathrm{~h}$ at $4{ }^{\circ} \mathrm{C}$ and $230 \mathrm{~V}$ in $0.5 \times \mathrm{TB}$ buffer. ${ }^{32} \mathrm{P}$ was imaged using the Personal Molecular Imager (Bio-Rad), and band intensities were quantified with the Quantity One software (Bio-Rad).

\section{MST}

Brat-NHL was labeled using the Monolith NT protein-labeling kit RED-NHS (NanoTemper Technologies) according to the manufacturer's instructions except that the time of incubation with the reactive dye was reduced to $20 \mathrm{~min}$. Thermophoresis experiments were carried out with $50 \mathrm{nM}$ labeled Brat-NHL in 20 $\mathrm{mM}$ Tris (pH 8), $150 \mathrm{mM} \mathrm{NaCl}, 0.2 \mathrm{mg} / \mathrm{mL}$ BSA, $0.05 \%$ Tween 20, and $0.2 \mathrm{mg} / \mathrm{mL}$ yeast tRNA (Ambion) with the indicated RNA concentrations at $80 \%$ MST power and $20 \%$ LED power in hydrophilic capillaries on a Monolith NT.115 at $20^{\circ} \mathrm{C}$ (NanoTemper Technologies). The recorded fluorescence was normalized and processed using the KaleidaGraph 4.1 software and fitted using the quadratic fitting formular ( $K_{d}$ formular) derived from the law of mass action.

\section{Western blotting}

To estimate expression levels of HA fusion proteins or endogenous Brat, lysates in PLB were separated by SDS-PAGE and transferred to nitrocellulose membranes (GE Healthcare). Membranes were probed with anti-HA, (1:1000; 16B12, Covance), anti- $\alpha$-tubulin (1:10.000; DM1A, Sigma), or anti-Brat 3A9 (1:500, a kind gift of Y. Zhang) (Shi et al. 2013).

\section{Acknowledgments}

We are grateful to Sigrun Ammon, Corinna Friederich, Kevin Schall, and Uwe Plessmann for excellent technical assistance; Anne Dueck, Melina Musri, and Jan Medenbach for discussion; and Marina Chekulaeva, Jan Medenbach, and Yong Zhang for reagents. This work was supported in part by grants from the Deutsche Forschungsgemeinschaft (SFB 960 to G.M., and SFB 860 to H.U.), the European Research Council (ERC grant "sRNAs" to G.M.), the Bavarian Genome Research Network (BayGene to G.M.), and the Bavarian Biosystems Network (BioSysNet to G.M.). J.H. received post-doctoral fellowships from the Swedish Research Council (Vetenskapsrådet) and the European Molecular Biology Organization (grant ALTF276-2010).

\section{References}

Arama E, Dickman D, Kimchie Z, Shearn A, Lev Z. 2000. Mutations in the $\beta$-propeller domain of the Drosophila brain tumor (brat) protein induce neoplasm in the larval brain. Oncogene 19: 3706-3716.

Asaoka-Taguchi M, Yamada M, Nakamura A, Hanyu K, Kobayashi S. 1999. Maternal Pumilio acts together with Nanos in germline development in Drosophila embryos. Nat Cell Biol 1: 431-437.

Baker RT, Catanzariti AM, Karunasekara Y, Soboleva TA, Sharwood R, Whitney S, Board PG. 2005. Using deubiquitylating enzymes as research tools. Methods Enzymol 398: 540-554.

Baltz AG, Munschauer M, Schwanhausser B, Vasile A, Murakawa Y, Schueler M, Youngs N, Penfold-Brown D, Drew K, Milek M, et al. 2012. The mRNA-bound proteome and its global occupancy profile on protein-coding transcripts. Mol Cell 46: 674-690.

Betschinger J, Mechtler K, Knoblich JA. 2006. Asymmetric segregation of the tumor suppressor brat regulates self-renewal in Drosophila neural stem cells. Cell 124: 1241-1253.

Castello A, Fischer B, Eichelbaum K, Horos R, Beckmann BM, Strein C, Davey NE, Humphreys DT, Preiss T, Steinmetz LM, et al. 2012. Insights into RNA biology from an atlas of mammalian mRNA-binding proteins. Cell 149: 1393-1406.

Catanzariti AM, Soboleva TA, Jans DA, Board PG, Baker RT. 2004. An efficient system for high-level expression and easy purification of authentic recombinant proteins. Protein Sci 13: 1331-1339.

Chang HM, Martinez NJ, Thornton JE, Hagan JP, Nguyen KD, Gregory RI. 2012. Trim71 cooperates with microRNAs to repress Cdkn1a expression and promote embryonic stem cell proliferation. Nat Commun 3: 923.

Chekulaeva M, Filipowicz W, Parker R. 2009. Multiple independent domains of dGW182 function in miRNA-mediated repression in Drosophila. RNA 15: 794-803.

Cherbas L, Willingham A, Zhang D, Yang L, Zou Y, Eads BD, Carlson JW, Landolin JM, Kapranov P, Dumais J, et al. 2011. The transcriptional diversity of 25 Drosophila cell lines. Genome Res 21: 301-314. 
Cho PF, Gamberi C, Cho-Park YA, Cho-Park IB, Lasko P, Sonenberg N. 2006. Cap-dependent translational inhibition establishes two opposing morphogen gradients in Drosophila embryos. Curr Biol 16: 2035-2041.

Chufan EE, De M, Eipper BA, Mains RE, Amzel LM. 2009. Amidation of bioactive peptides: the structure of the lyase domain of the amidating enzyme. Structure 17: 965-973.

Curtis D, Lehmann R, Zamore PD. 1995. Translational regulation in development. Cell 81: 171-178.

Duchaine TF, Wohlschlegel JA, Kennedy S, Bei Y, Conte D Jr, Pang K, Brownell DR, Harding S, Mitani S, Ruvkun G, et al. 2006. Functional proteomics reveals the biochemical niche of C. elegans DCR-1 in multiple small-RNA-mediated pathways. Cell 124: 343-354.

Edwards TA, Wilkinson BD, Wharton RP, Aggarwal AK. 2003. Model of the brain tumor-Pumilio translation repressor complex. Genes Dev 17: 2508-2513.

Frank DJ, Edgar BA, Roth MB. 2002. The Drosophila melanogaster gene brain tumor negatively regulates cell growth and ribosomal RNA synthesis. Development 129: 399-407.

Gerber AP, Luschnig S, Krasnow MA, Brown PO, Herschlag D. 2006. Genome-wide identification of mRNAs associated with the translational regulator PUMILIO in Drosophila melanogaster. Proc Natl Acad Sci 103: 4487-4492.

Good MC, Greenstein AE, Young TA, Ng HL, Alber T. 2004. Sensor domain of the Mycobacterium tuberculosis receptor Ser/Thr protein kinase, $\mathrm{PknD}$, forms a highly symmetric $\beta$ propeller. J Mol Biol 339: 459-469.

Hammell CM, Lubin I, Boag PR, Blackwell TK, Ambros V. 2009. nhl-2 modulates microRNA activity in Caenorhabditis elegans. Cell 136: 926-938.

Harris RE, Pargett M, Sutcliffe C, Umulis D, Ashe HL. 2011. Brat promotes stem cell differentiation via control of a bistable switch that restricts BMP signaling. Dev Cell 20: 72-83.

Hulskamp M, Pfeifle C, Tautz D. 1990. A morphogenetic gradient of hunchback protein organizes the expression of the gap genes Kruppel and knirps in the early Drosophila embryo. Nature 346: 577-580.

Jerabek-Willemsen M, Wienken CJ, Braun D, Baaske P, Duhr S. 2011. Molecular interaction studies using microscale thermophoresis. Assay Drug Dev Technol 9: 342-353.

Kanai Y, Dohmae N, Hirokawa N. 2004. Kinesin transports RNA: isolation and characterization of an RNA-transporting granule. Neuron 43: 513-525.

Kramer K, Hummel P, Hsiao H-H, Luo X, Wahl M, Urlaub H. 2011. Mass-spectrometric analysis of proteins cross-linked to 4-thio-uracil- and 5-bromo-uracil-substituted RNA. Int $I$ Mass Spectrom 304: 184-194.

Kwon SC, Yi H, Eichelbaum K, Fohr S, Fischer B, You KT, Castello A, Krijgsveld J, Hentze MW, Kim VN. 2013. The RNA-binding protein repertoire of embryonic stem cells. Nat Struct Mol Biol 20: 1122-1130.

Lau CK, Bachorik JL, Dreyfuss G. 2009. Gemin5-snRNA interaction reveals an RNA binding function for WD repeat domains. Nat Struct Mol Biol 16: 486-491.

Lee CY, Wilkinson BD, Siegrist SE, Wharton RP, Doe CQ. 2006. Brat is a Miranda cargo protein that promotes neuronal differentiation and inhibits neuroblast self-renewal. Dev Cell 10: 441-449.

Li Y, Maines JZ, Tastan OY, McKearin DM, Buszczak M. 2012. Mei-P26 regulates the maintenance of ovarian germline stem cells by promoting BMP signaling. Development 139: 15471556.

Li Y, Zhang Q, Carreira-Rosario A, Maines JZ, McKearin DM, Buszczak M. 2013. Mei-p26 cooperates with Bam, Bgen and
Sxl to promote early germline development in the Drosophila ovary. PLOS ONE 8: e58301.

Loedige I, Gaidatzis D, Sack R, Meister G, Filipowicz W. 2013. The mammalian TRIM-NHL protein TRIM71/LIN-41 is a repressor of mRNA function. Nucleic Acids Res 41: 518-532.

Luo X, Hsiao HH, Bubunenko M, Weber G, Court DL, Gottesman ME, Urlaub H, Wahl MC. 2008. Structural and functional analysis of the E. coli NusB-S10 transcription antitermination complex. Mol Cell 32: 791-802.

Muraro NI, Weston AJ, Gerber AP, Luschnig S, Moffat KG, Baines RA. 2008. Pumilio binds para mRNA and requires Nanos and Brat to regulate sodium current in Drosophila motoneurons. J Neurosci 28: 2099-2109.

Murata Y, Wharton RP. 1995. Binding of pumilio to maternal hunchback mRNA is required for posterior patterning in Drosophila embryos. Cell 80: 747-756.

Neumuller RA, Betschinger I, Fischer A, Bushati N, Poernbacher I, Mechtler K, Cohen SM, Knoblich JA. 2008. Mei-P26 regulates microRNAs and cell growth in the Drosophila ovarian stem cell lineage. Nature 454: 241-245.

Nusslein-Volhard C, Frohnhofer HG, Lehmann R. 1987. Determination of anteroposterior polarity in Drosophila. Science 238: 1675-1681.

Reymond A, Meroni G, Fantozzi A, Merla G, Cairo S, Luzi L, Riganelli D, Zanaria E, Messali S, Cainarca S, et al. 2001. The tripartite motif family identifies cell compartments. EMBO I 20: $2140-2151$.

Rybak A, Fuchs H, Hadian K, Smirnova L, Wulczyn EA, Michel G, Nitsch R, Krappmann D, Wulczyn FG. 2009. The let-7 target gene mouse lin-41 is a stem cell specific E3 ubiquitin ligase for the miRNA pathway protein Ago2. Nat Cell Biol 11: $1411-1420$

Sardiello M, Cairo S, Fontanella B, Ballabio A, Meroni G. 2008. Genomic analysis of the TRIM family reveals two groups of genes with distinct evolutionary properties. BMC Evol Biol 8: 225 .

Schwamborn JC, Berezikov E, Knoblich JA. 2009. The TRIMNHL protein TRIM32 activates microRNAs and prevents self-renewal in mouse neural progenitors. Cell 136: 913925.

Scrima A, Konickova R, Czyzewski BK, Kawasaki Y, Jeffrey PD, Groisman R, Nakatani Y, Iwai S, Pavletich NP, Thoma NH. 2008. Structural basis of UV DNA-damage recognition by the DDB1-DDB2 complex. Cell 135: 1213-1223.

Shi W, Chen Y, Gan G, Wang D, Ren J, Wang Q, Xu Z, Xie W, Zhang YQ. 2013. Brain tumor regulates neuromuscular synapse growth and endocytosis in Drosophila by suppressing mad expression. J Neurosci 33: 12352-12363.

Slack FJ, Ruvkun G. 1998. A novel repeat domain that is often associated with RING finger and B-box motifs. Trends Biochem Sci 23: 474-475.

Slack FJ, Basson M, Liu Z, Ambros V, Horvitz HR, Ruvkun G. 2000. The lin-41 RBCC gene acts in the C. elegans heterochronic pathway between the let-7 regulatory RNA and the LIN-29 transcription factor. Mol Cell 5: 659-669.

Sonoda J, Wharton RP. 1999. Recruitment of Nanos to hunchback mRNA by Pumilio. Genes Dev 13: 2704-2712.

Sonoda J, Wharton RP. 2001. Drosophila brain tumor is a translational repressor. Genes Dev 15: 762-773.

Stirnimann CU, Petsalaki E, Russell RB, Müller CW. 2010. WD40 proteins propel cellular networks. Trends Biochem Sci 35: 565-574.

Tautz D, Pfeifle C. 1989. A non-radioactive in situ hybridization method for the localization of specific RNAs in Drosophila embryos reveals translational control of the segmentation gene hunchback. Chromosoma 98: 81-85. 
Loedige et al.

Wang X, McLachlan J, Zamore PD, Hall TM. 2002. Modular recognition of RNA by a human pumilio-homology domain. Cell 110: 501-512.

Weidmann CA, Goldstrohm AC. 2012. Drosophila Pumilio protein contains multiple autonomous repression domains that regulate mRNAs independently of Nanos and brain tumor. Mol Cell Biol 32: 527-540.

Wharton RP, Struhl G. 1991. RNA regulatory elements mediate control of Drosophila body pattern by the posterior morphogen nanos. Cell 67: 955-967.

Worringer KA, Rand TA, Hayashi Y, Sami S, Takahashi K, Tanabe K, Narita M, Srivastava D, Yamanaka S. 2014. The let-7/LIN-41 pathway regulates reprogramming to human induced pluripotent stem cells by controlling expression of prodifferentiation genes. Cell Stem Cell 14: 40-52.

Zamore PD, Williamson JR, Lehmann R. 1997. The Pumilio protein binds RNA through a conserved domain that defines a new class of RNA-binding proteins. RNA 3: 1421-1433.

Zamore PD, Bartel DP, Lehmann R, Williamson JR. 1999. The PUMILIO-RNA interaction: a single RNA-binding domain monomer recognizes a bipartite target sequence. Biochemistry 38: 596-604.

Zheng L, Baumann U, Reymond JL. 2004. An efficient one-step site-directed and site-saturation mutagenesis protocol. Nucleic Acids Res 32: e115.

Zillner K, Jerabek-Willemsen M, Duhr S, Braun D, Langst G, Baaske P. 2012. Microscale thermophoresis as a sensitive method to quantify protein: nucleic acid interactions in solution. Methods Mol Biol 815: 241-252. 


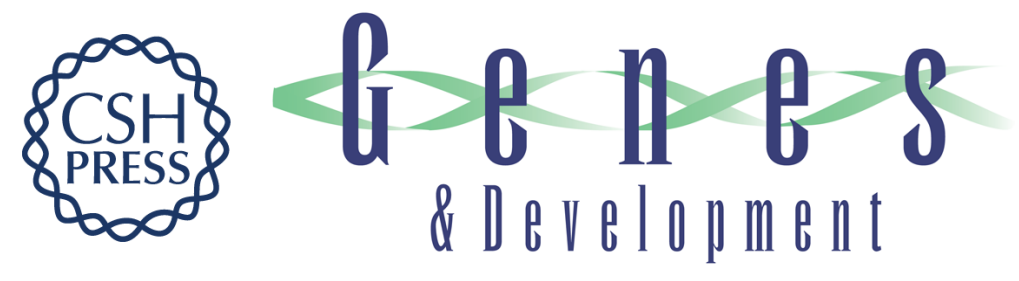

\section{The NHL domain of BRAT is an RNA-binding domain that directly contacts the hunchback mRNA for regulation}

Inga Loedige, Mathias Stotz, Saadia Qamar, et al.

Genes Dev. 2014, 28:

Access the most recent version at doi:10.1101/gad.236513.113

\section{Supplemental http://genesdev.cshlp.org/content/suppl/2014/04/02/28.7.749.DC1 \\ Material}

References This article cites 55 articles, 13 of which can be accessed free at:

http://genesdev.cshlp.org/content/28/7/749.full.html\#ref-list-1

Creative This article is distributed exclusively by Cold Spring Harbor Laboratory Press for the first

Commons six months after the full-issue publication date (see

License http://genesdev.cshlp.org/site/misc/terms.xhtml). After six months, it is available under a Creative Commons License (Attribution-NonCommercial 4.0 International), as described at http://creativecommons.org/licenses/by-nc/4.0/.

Email Alerting Receive free email alerts when new articles cite this article - sign up in the box at the top Service right corner of the article or click here.

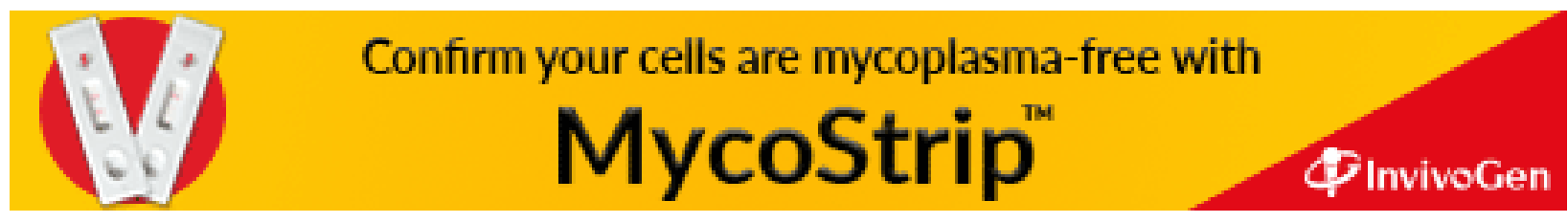

